\title{
Time Fractional Poisson Equations: Representations and Estimates
}

\author{
Zhen-Qing Chen Panki Kim Takashi Kumagai Jian Wang
}

\begin{abstract}
In this paper, we study existence and uniqueness of strong as well as weak solutions for general time fractional Poisson equations. We show that there is an integral representation of the solutions of time fractional Poisson equations with zero initial values in terms of semigroup for the infinitesimal spatial generator $\mathcal{L}$ and the corresponding subordinator associated with the time fractional derivative. This integral representation has an integral kernel $q(t, x, y)$, which we call the fundamental solution for the time fractional Poisson equation, if the semigroup for $\mathcal{L}$ has an integral kernel. We further show that $q(t, x, y)$ can be expressed as a time fractional derivative of the fundamental solution for the homogenous time fractional equation under the assumption that the associated subordinator admits a conjugate subordinator. Moreover, when the Laplace exponent of the associated subordinator satisfies the weak scaling property and its distribution is self-decomposable, we establish two-sided estimates for the fundamental solution $q(t, x, y)$ through explicit estimates of transition density functions of subordinators.
\end{abstract}

AMS 2010 Mathematics Subject Classification: Primary 26A33, 60H30; Secondary: 34K37

Keywords and phrases: time fractional derivative, Caputo derivative, Poisson equation, subordinator, inverse subordinator, conjugate subordinator, fundamental solution

\section{Introduction}

In recent years, linear as well as non-linear partial differential equations with fractional derivatives are extensively studied. This interest has been stimulated by anomalous diffusion phenomena appeared in diverse fields including mathematics, physics, chemistry, engineering, biology, geophysics and hydrology, see [30, 32, 33, 41, 42, and the references therein. The present paper is concerned with solutions of general time fractional Poisson equations.

For a given function $w:(0, \infty) \rightarrow[0, \infty)$ that is unbounded, non-increasing and having $\int_{0}^{\infty} \min \{1, x\}(-d w(x))<\infty$, a generalized time fractional derivative with weight $w$ is defined by

$$
\partial_{t}^{w} f(t):=\frac{d}{d t} \int_{0}^{t} w(t-s)(f(s)-f(0)) d s,
$$

whenever the right hand side is well defined. See [8, 13, 24, 27]. In particular, when $w(s)=$ $\frac{1}{\Gamma(1-\beta)} s^{-\beta}$ for $\beta \in(0,1)$ (where $\Gamma(t)=\int_{0}^{\infty} s^{t-1} e^{-s} d s$ is the Gamma function), $\partial_{t}^{w} f$ is just the 
Caputo derivative of order $\beta$, i.e.,

$$
\partial_{t}^{\beta} f(t):=\frac{1}{\Gamma(1-\beta)} \frac{d}{d t} \int_{0}^{t}(t-s)^{-\beta}(f(s)-f(0)) d s .
$$

Let $\mathcal{L}$ be a linear (can be unbounded) operator on some function space over a locally compact separable metric space $E$ with a fully-supported Radon measure $\mu$. We consider the following general time fractional Poisson equation

$$
\partial_{t}^{w} u(\cdot, x)(t)=\mathcal{L} u(t, \cdot)(x)+f(t, x) \quad t \in\left(0, T_{0}\right], x \in E .
$$

where $T_{0}>0$ and $f(t, x)$ is a given function on $\left(0, T_{0}\right] \times E$. For simplicity, in the following we will write the equation in (1.2) as

$$
\partial_{t}^{w} u(t, x)=\mathcal{L} u(t, x)+f(t, x) .
$$

When $f(t, x)=0,(1.2)$ is reduced into the following general time fractional Cauchy equation

$$
\partial_{t}^{w} u(t, x)=\mathcal{L} u(t, x), \quad t \in\left(0, T_{0}\right], x \in E .
$$

Solutions of time fractional Cauchy as well as Poisson equations have been attracted a lot of attentions in the community of analysis, PDEs and stochastic analysis, see [1, 6, 12, 15, 17, 18, 28, 31, 38, 39] and the references therein. We note that most of quoted papers are concentrated on the Caputo derivative of fractional order. Recently, Chen [8] studied equation (1.3) with $T_{0}=\infty$ for any unbounded and non-increasing function $w$ with $\int_{0}^{\infty} \min \{1, x\}(-d w(x))<\infty$ and for any infinitesimal generator $\mathcal{L}$ that generates a uniformly bounded and strongly continuous semigroup in some Banach space, and obtained strong existence and uniqueness of solutions to (1.3). The main feature of the approach in [8] is a detailed analysis of the subordinator associated with the function $w$ together with a probabilistic representation of the solution. The existence and uniqueness results in [8] for strong solutions of (1.3) have been extended to that of weak solutions in [13] when the infinitesimal generator $\mathcal{L}$ is a symmetric operator in some $L^{2}$ space with $T_{0}=\infty$. Moreover, twosided estimates on the fundamental solution of (1.3) have been derived in [13] for a family of $w$ and $\mathcal{L}$.

In this paper, we call $q(t, x, y)$ the fundamental solution to the time fractional Poisson equation (1.2) (with $u(0, x)=0$ ), and call $p(t, x, y)$ the fundamental solution to the homogenous time fractional equation (1.3).

The aim of our paper is twofold. One is to study existence and uniqueness of strong as well as weak solutions for (1.2) and to derive an integral representation of the solution to the time fractional Poisson equation (1.2) with zero initial data in terms of the semigroup $\left\{P_{t}^{0} ; t \geq 0\right\}$ of $\mathcal{L}$ and the density $\bar{p}(r, t)$ of the driftless subordinator $S_{r}$ whose Lévy measure is $\nu$ and $w(x)=\nu(x, \infty)$ is the non-increasing function $w$ in (1.1). When $\left\{P_{t}^{0} ; t \geq 0\right\}$ has an integral kernel $p^{0}(t, x, y)$, the integral representation obtained in this paper for the solution of (1.2) admits an integral kernel $q(t, x, y)$, which we call the the fundamental solution to the time fractional Poisson equation (1.2) (with $u(0, x)=0)$. The other is to establish two-sided estimates on $q(t, x, y)$. Our approach is mainly based on probabilistic ideas combined with some analytic techniques. The paper can be viewed as a sequel to [8, 13, where existence and uniqueness, probabilistic representations as well as two-sided estimates for the fundamental solution to the homogenous time fractional equation 
(1.3) are given. In particular, it is shown in [8] that $p(t, x, y):=\mathbb{E}\left[p^{0}\left(E_{t}, x, y\right)\right]$, where $E_{t}$ is the inverse of the subordinator $S$, is the fundamental solution for the time-homogenous time fractional equation (1.2). However, the study of the fundamental solution $q(t, x y)$ of (1.2) is harder than the fundamental solution $p(t, x, y)$ of the equation (1.3). To obtain two-sided estimates for the fundamental solution $q(t, x, y)$ for the time fractional Poisson equation, we also need some explicit estimates for transition density functions of subordinators, which are highly non-trivial.

Two recent papers [25, 37] considered a slightly different form of time fractional derivative but have a probabilistic representation analogous to us. The proofs there are different from ours and scope of that two papers are restricted to Feller generators in Euclidean spaces. See also [14] for a recently related work where the time fractional derivative having a possibly time-dependent kernel and the spatial operator is a Dirichlet Laplacian in a regular Euclidean domain.

We now present the precise setting of this paper. Throughout this paper, let $S=\left\{S_{t}, \mathbb{P} ; t \geq 0\right\}$ be a driftless subordinator with $S_{0}=0$ that has a bounded density $\bar{p}(t, \cdot)$ for every $t>0$. Denote by $\phi$ the Laplace exponent of $S$; that is,

$$
\mathbb{E}\left[e^{-\lambda S_{t}}\right]=e^{-t \phi(\lambda)}, \quad \lambda>0, t \geq 0 .
$$

The Laplace exponent $\phi$ of $S$ is also called Bernstein function (vanishes at the origin) in the literature. Since $S$ has no drift, it is well known that there is a unique measure $\nu$ on $(0, \infty)$, which is called Lévy measure of $S$, satisfying $\int_{0}^{\infty} \min \{1, x\} \nu(d x)<\infty$ such that

$$
\phi(\lambda)=\int_{0}^{\infty}\left(1-e^{-\lambda x}\right) \nu(d x) .
$$

Let

$$
w(x):=\nu(x, \infty)
$$

We always assume that the Lévy measure $\nu$ of the subordinator $S$ is infinite, which is equivalent to $w(x)$ being unbounded. (However, $w$ is always locally integrable on $[0, \infty)$; see [8, (2.2)].) Let $(\mathcal{L}, \mathcal{D}(\mathcal{L}))$ be an infinitesimal generator corresponding to a uniformly bounded and strongly continuous semigroup $\left\{P_{t}^{0} ; t \geq 0\right\}$ in some Banach space. In this paper, we are concerned with time fractional Poisson equation (1.2) with the weight function $w(x)$ and the infinitesimal generator $(\mathcal{L}, \mathcal{D}(\mathcal{L}))$.

We now state the existence and uniqueness for the strong as well as weak solutions of time fractional heat equations, which is one of the main results of this paper. Define for $t>0, E_{t}=$ $\inf \left\{s>0: S_{s}>t\right\}$, the inverse subordinator.

Theorem 1.1 (Strong solution) Assume that the subordinator $\left\{S_{t}, \mathbb{P} ; t \geq 0\right\}$ has a bounded density $\bar{p}(t, \cdot)$ for each $t>0$ and its Lévy measure $\nu$ is infinite. Suppose that $\left\{P_{t}^{0} ; t \geq 0\right\}$ is a uniformly bounded and strongly continuous semigroup in some Banach space $(\mathbb{B},\|\cdot\|)$ over a locally compact separable metric space $E$ and $(\mathcal{L}, \mathcal{D}(\mathcal{L}))$ is its infinitesimal generator. Let $T_{0} \in(0, \infty)$, and $g \in \mathcal{D}(\mathcal{L})$ and $f(t, x)$ be a function defined on $\left(0, T_{0}\right] \times E$ so that for a.e. $t \in\left(0, T_{0}\right], f(t, \cdot) \in \mathcal{D}(\mathcal{L})$ and

$$
\int_{0}^{T_{0}}\|\mathcal{L} f(t, \cdot)\| d t<\infty \quad \text { and } \quad\|f(t, \cdot)\| \leq K<\infty \text { for a.e. } t \in\left(0, T_{0}\right] .
$$


Then the function

$$
\begin{aligned}
u(t, x): & =\mathbb{E}\left[P_{E_{t}}^{0} g(x)\right]+\mathbb{E}\left[\int_{r=0}^{\infty} \mathbf{1}_{\left\{S_{r}<t\right\}} P_{r}^{0} f\left(t-S_{r}, \cdot\right)(x) d r\right] \\
& =\mathbb{E}\left[P_{E_{t}}^{0} g(x)\right]+\int_{s=0}^{t} \int_{r=0}^{\infty} P_{r}^{0} f(s, \cdot)(x) \bar{p}(r, t-s) d r d s
\end{aligned}
$$

is the unique (strong) solution of $\partial_{t}^{w} u(t, x)=\mathcal{L} u(t, x)+f(t, x)$ on $\left(0, T_{0}\right] \times E$ with $u(0, x)=g(x)$ in the following sense:

(i) $u(t, \cdot)$ is well defined as an element in $\mathbb{B}$ for each $t \in\left(0, T_{0}\right]$ such that $\sup _{t \in\left(0, T_{0}\right]}\|u(t, \cdot)\|<\infty$, $t \mapsto u(t, x)$ is continuous in $(\mathbb{B},\|\cdot\|)$ and $\lim _{t \rightarrow 0}\|u(t, \cdot)-g(\cdot)\|=0$.

(ii) For a.e. $t \in\left(0, T_{0}\right], u(t, \cdot) \in \mathcal{D}(\mathcal{L})$ and $\mathcal{L} u(t, \cdot)$ exists in the Banach space $\mathbb{B}$ such that $\int_{0}^{T_{0}}\|\mathcal{L} u(t, \cdot)\| d t<\infty$.

(iii) For every $T \in\left(0, T_{0}\right], \int_{0}^{T} w(T-t)(u(t, x)-g(x)) d t$ is absolutely convergent in $(\mathbb{B},\|\cdot\|)$ and

$$
\int_{0}^{T} w(T-t)(u(t, \cdot)-g(\cdot)) d t=\int_{0}^{T}(f(t, \cdot)+\mathcal{L} u(t, \cdot)) d t \quad \text { in } \mathbb{B} .
$$

Remark 1.2 (i) Clearly by Theorem 1.1, if condition (1.5) holds for every $T_{0}>0$, then the function $u(t, x)$ given by (1.6) is well defined for all $t>0$ and is the unique strong solution of $\partial_{t}^{w} u(t, x)=\mathcal{L} u(t, x)+f(t, x)$ on $(0, \infty) \times E$ with $u(0, x)=g(x)$. Similar remark applies to Theorem 1.3 below on weak solutions as well.

(ii) When $f=0$, the above result is established in [8, Theorem 1.1]. We point out that in 8 , Theorem 2.3] (where $f=0$ ), the continuity of $t \mapsto \mathcal{L} u(t, \cdot)$ in $(\mathbb{B},\|\cdot\|)$ appears as a part of the definition of the strong solution. But one can see from the uniqueness part of the proof of $[8$, Theorem 2.3] that the continuity of $t \mapsto \mathcal{L} u(t, \cdot)$ in $(\mathbb{B},\|\cdot\|)$ is not used. Thus the continuity of $t \mapsto \mathcal{L} u(t, \cdot)$ should be one of properties of the strong solution there, and can be removed from the definition of the strong solution in the statement of [8, Theorem 2.3].

Note that, if we extend the definition of $f(t, \cdot)$ to $\left(-\infty, T_{0}\right]$ by letting $f(t, \cdot) \equiv 0$ for $t \leq 0$, then (1.6) can be rewritten as

$$
u(t, x)=\mathbb{E}\left[P_{E_{t}}^{0} g(x)\right]+\mathbb{E}\left[\int_{r=0}^{\infty} P_{r}^{0} f\left(t-S_{r}, \cdot\right)(x) d r\right] .
$$

(Cf., [25, (35)] and [37, (1.3)].)

Theorem 1.3 (Weak solution) Assume that the subordinator $\left\{S_{t}, \mathbb{P} ; t \geq 0\right\}$ has a bounded density $\bar{p}(t, \cdot)$ for each $t>0$ and its Lévy measure $\nu$ is infinite. Suppose that $E$ is a locally compact separable metric space and that $\mu$ is a $\sigma$-finite Radon measure on $E$ with full support. Suppose that $(\mathcal{L}, \mathcal{D}(\mathcal{L}))$ is the infinitesimal generator of a Dirichlet form on $L^{2}(E ; \mu)$ and $\left\{P_{t}^{0} ; t \geq 0\right\}$ is its 
associated transition semigroup in $L^{2}(E ; \mu)$. Denote by $\langle\cdot, \cdot\rangle$ the inner product of $L^{2}(E ; \mu)$. Suppose that $T_{0} \in(0, \infty)$, and $g \in L^{2}(E ; \mu)$ and that $f(t, x)$ is a function on $\left(0, T_{0}\right] \times E$ so that

$$
\|f(t, \cdot)\|_{L^{2}(E ; \mu)} \leq K<\infty \text { for a.e. } t \in\left(0, T_{0}\right] .
$$

Then $u(t, x)$ defined by (1.6) is the unique weak solution of $\partial_{t}^{w} u(t, x)=\mathcal{L} u(t, x)+f(t, x)$ on $\left(0, T_{0}\right] \times E$ with $u(0, x)=g(x)$ in the following sense:

(i) $u(t, \cdot)$ is well defined as an element in $L^{2}(E ; \mu)$ for every $t \in\left(0, T_{0}\right]$ satisfying that $t \mapsto u(t, x)$ is continuous in $L^{2}(E ; \mu)$,

$$
\sup _{t \in\left(0, T_{0}\right]}\|u(t, \cdot)\|_{L^{2}(E ; \mu)}<\infty \quad \text { and } \quad u(t, \cdot) \rightarrow g \text { in } L^{2}(E ; \mu) \text { as } t \rightarrow 0 \text {. }
$$

(ii) For every $T \in\left(0, T_{0}\right], \int_{0}^{T} w(T-t)(u(t, x)-g(x)) d t$ is absolutely convergent in $L^{2}(E ; \mu)$, and

$$
\left\langle\int_{0}^{T} w(T-s)(u(s, \cdot)-g(\cdot)) d s, \varphi\right\rangle=\int_{0}^{T}\langle f(s, \cdot), \varphi\rangle d s+\int_{0}^{T}\langle u(s, \cdot), \mathcal{L} \varphi\rangle d s
$$

for every $\varphi \in \mathcal{D}(\mathcal{L})$.

Furthermore, if $\sup _{t \in\left(0, T_{0}\right]}\|\mathcal{L} f(t, \cdot)\|<\infty$, then $t \mapsto \mathcal{L} u(t, x)$ is also continuous in $L^{2}(E ; \mu)$.

A direct but important consequence of Theorems 1.1 and 1.3 is that, when the semigroup $\left\{P_{t}^{0} ; t \geq 0\right\}$ has a density function $p^{0}(t, x, y)$, the fundamental solution $q(t, x, y)$ for the time fractional Poisson equation is

$$
q(t, x, y):=\int_{0}^{\infty} p^{0}(r, x, y) \bar{p}(r, t) d r
$$

Formula (1.9) clearly implies the positivity of $q(t, x, y)$. Furthermore, (1.9) enables us to establish in Section 4 two-sided estimates for the fundamental solution $q(t, x, y)$ for the time fractional Poisson equation using estimates of $p^{0}(r, x, y)$ and $\bar{p}(r, t)$ only.

Throughout the paper, we write $h(s) \simeq f(s)$, if there exist constants $c_{1}, c_{2}>0$ such that $c_{1} f(s) \leq h(s) \leq c_{2} f(s)$ for the specified range of the argument $s$. Similarly, we write $h(s) \asymp$ $f(s) g(s)$, if there exist constants $C_{1}, c_{1}, C_{2}, c_{2}>0$ such that $f\left(C_{1} s\right) g\left(c_{1} s\right) \leq h(s) \leq f\left(C_{2} s\right) g\left(c_{2} s\right)$ for the specified range of $s$.

The following theorem is a special case of Theorems 4.8 and 4.9, Suppose that the fundamental solution $p^{0}(t, x, y)$ of $\mathcal{L}$ admits the following two-sided estimates:

$$
p^{0}(t, x, y) \asymp t^{-d / \alpha} F\left(d(x, y) / t^{1 / \alpha}\right),
$$

where either (i) $F(r)=\exp \left(-r^{\alpha /(\alpha-1)}\right)$ for $\alpha \geq 2$; or (ii) $F(r)=(1+r)^{-d-\alpha}$ with $\alpha>0$. Note that for case (i), a priori $\alpha>1$ but in fact such estimates can hold only if $\alpha \geq 2$ (see 4, Page 1644]). Case (i) typically corresponds to diffusion processes. When

$$
\mathcal{L}=\sum_{i, j=1}^{d} \frac{\partial}{\partial x_{i}}\left(a_{i j}(x) \frac{\partial}{\partial x_{j}}\right) \quad \text { with } \quad \lambda^{-1} I_{d \times d} \leq\left(a_{i j}(x)\right) \leq \lambda I_{d \times d} \text { on } \mathbb{R}^{d},
$$


where $I_{d \times d}$ denotes the $d \times d$ identity matrix, it is known due to a result by Aronson [2] that $\mathcal{L}$ admits such estimates with $\alpha=2$. When $\mathcal{L}$ is the Laplacian on a two-dimensional unbounded Sierpinski gasket, it is shown by Barlow and Perkins in [5] that the two-sided heat kernel estimates (1.10) of case (i) hold $d=\log 3 / \log 2$ and $\alpha=d_{w}:=\log 5 / \log 2$. Case (ii) typically corresponds to pure jump processes. It is shown in [9] that estimates (1.10) of case (ii) hold for symmetric $\alpha$-stable-like process on Alfhors $d$-regular space $E$ for $0<\alpha<2$. See [11] for examples with $\alpha \geq 2$.

For $\beta \in(0,1)$, define

$$
\begin{aligned}
& H_{\leq 1}(t, r)= \begin{cases}t^{\beta-1-\beta d / \alpha}, & \text { if } d<2 \alpha, \\
t^{-1-\beta} \log \left(\frac{2 t^{\beta}}{r^{\alpha}}\right), & \text { if } d=2 \alpha, \\
t^{-1-\beta} / r^{d-2 \alpha}, & \text { if } d>2 \alpha,\end{cases} \\
& H_{\geq 1}^{(j)}(t, r)=t^{2 \beta-1} / r^{d+\alpha}, \\
& H_{\geq 1}^{(c)}(t, r)=t^{\beta-1-\beta d / \alpha} \exp \left(-\left(r^{\alpha} / t^{\beta}\right)^{1 /(\alpha-\beta)}\right) .
\end{aligned}
$$

Theorem 1.4 Suppose that $\mathcal{L}$ is the generator of a Markov process and its fundamental solution $p^{0}(t, x, y)$ admits the two-sided estimates (1.10), and that $\left\{S_{t}, \mathbb{P} ; t \geq 0\right\}$ is a $\beta$-stable subordinator with $0<\beta<1$.

(i) Suppose $F(r)=(1+r)^{-d-\alpha}$ with $\alpha>0$. Then the fundamental solution $q(t, x, y)$ for the time fractional Poisson equation (1.2) satisfies

$$
q(t, x, y) \simeq \begin{cases}H_{\leq 1}(t, d(x, y)) & \text { if } d(x, y) \leq t^{\beta / \alpha} \\ H_{\geq 1}^{(j)}(t, d(x, y)) & \text { if } d(x, y) \geq t^{\beta / \alpha}\end{cases}
$$

(ii) Suppose $F(r)=\exp \left(-r^{\alpha /(\alpha-1)}\right)$ with $\alpha \geq 2$. Then the fundamental solution $q(t, x, y)$ for the time fractional Poisson equation (1.2) satisfies

$$
\begin{array}{ll}
q(t, x, y) \simeq H_{\leq 1}(t, d(x, y)) & \text { if } d(x, y) \leq t^{\beta / \alpha}, \\
q(t, x, y) \asymp H_{\geq 1}^{(c)}(t, d(x, y)) & \text { if } d(x, y) \geq t^{\beta / \alpha} .
\end{array}
$$

The rest of the paper is organized as follows. Existence and uniqueness of strong as well as weak solutions to the equation (1.2) are obtained in Section 2, Assuming further that $P_{t}^{0}$ has a density function $p^{0}(t, x, y)$ with respect to $\mu$, and that the subordinator $S$ is a special subordinator, we prove in Section 3 that the fundamental solution $q(t, x, y)$ for the time fractional Poisson equation and the fundamental solution $p(r, x, y)$ of the homogenous time fractional equation enjoy the following relation

$$
q(t, x, y)=\partial_{t}^{w^{*}} p(\cdot, x, y)(t) \quad \text { for a.e. } t>0 \text { and } \mu \text {-a.e. } x \neq y \text {. }
$$

where $w^{*}(x):=\nu^{*}(x, \infty)$ and $\nu^{*}$ is the Lévy measure of the conjugate subordinator $S^{*}$ to $S$. When $\partial_{t}^{w}$ is the Caputo derivative of order $\beta \in(0,1)$, i.e., $w(s)=\frac{1}{\Gamma(1-\beta)} s^{-\beta}$, its corresponding subordinator is the $\beta$-stable subordinator which has $(1-\beta)$-stable subordinator as its conjugate. Hence $\partial_{t}^{w^{*}}$ is the Caputo derivative of order $1-\beta$. In this case, that $\partial_{t}^{1-\beta} p(\cdot, x, y)(t)$ is the 
fundamental solution for (1.2) has been established in [15, 16, 38, 39] in some special cases of $\mathcal{L}$, and is called Duhamel's principle in some literature.

In Section 4, assuming that the distribution of subordinator is self-decomposable, we derive sharp estimates for the transition density function of the subordinator, which is of independent interest itself. Note that the self-decomposable distribution naturally occurs in scaling limits of random walks with random waiting times. See [29, Corollary 3.8]. Using estimates for the transition density function of the subordinator, we establish two-sided estimates for $q(t, x, y)$. Full proofs of the two-sided estimates for $q(t, x, y)$ is given in the appendix of this paper.

Throughout the paper, for $a, b \in \mathbb{R}$ we denote $a \wedge b:=\min \{a, b\}$ and $a \vee b:=\max \{a, b\}$. For any measurable function $f$ we denote $f^{+}:=f \vee 0$. For a Banach space $(\mathbb{B},\|\cdot\|)$, we also use $\|T\|$ to denote the operator norm for a linear operator $T:(\mathbb{B},\|\cdot\|) \rightarrow(\mathbb{B},\|\cdot\|)$.

A constant $c$ (without subscripts) denotes a strictly positive constant, whose value is unimportant and which may change from line to line. Constants $c_{0}, c_{1}, c_{2}, \ldots$ with subscripts denote strictly positive constants, and the labeling of the constants starts anew in the statement of each result and the each step of its proof.

\section{Time fractional Poisson equations}

Recall that throughout this paper, $S=\left\{S_{t}, \mathbb{P} ; t \geq 0\right\}$ is a driftless subordinator with infinite Lévy measure $\nu$ and starting from 0 ; that is, $S_{0}=0$. Recall that $w(x):=\nu(x, \infty)$ and that $E_{t}=\inf \left\{s>0: S_{s}>t\right\}, t>0$, is the inverse subordinator of $S$. The assumption that the Lévy measure $\nu$ is infinite (which is equivalent to $w(x)$ being unbounded) excludes compound Poisson processes. Under this assumption, almost surely, $t \mapsto S_{t}$ is strictly increasing and hence $t \mapsto E_{t}$ is continuous. Throughout this paper, we also assume that the subordinator $S_{r}$ has a bounded density function $\bar{p}(r, \cdot)$ for each $r>0$. This assumption holds, for example, when Hartman and Wintner's condition is satisfied, that is,

$$
\lim _{s \rightarrow \infty} \frac{\phi(s)}{\ln (1+s)}=\lim _{s \rightarrow \infty} \frac{1}{\ln (1+s)} \int_{0}^{\infty}\left(1-e^{-s x}\right) \nu(d x)=\infty .
$$

See [23, (74) on page 287].

Since $\mathbb{P}\left(E_{t} \leq r\right)=\mathbb{P}\left(S_{r}>t\right)$, the following result is from [8].

Lemma 2.1 ([즈, Lemma 2.1]) There is a Borel set $\mathcal{N} \subset(0, \infty)$ having zero Lebesgue measure such that for every $t \in(0, \infty) \backslash \mathcal{N}$, the inverse subordinator $E_{t}$ has a density function given by

$$
\frac{d}{d r} \mathbb{P}\left(E_{t} \leq r\right)=\int_{0}^{t} w(t-s) \bar{p}(r, s) d s, \quad r>0 .
$$

Let

$$
G^{(S)}(t):=\int_{0}^{\infty} \bar{p}(r, t) d r
$$

be the potential density of the subordinator $S$, and denote by $G^{(S)}$ the 0 -order resolvent of $S$; that is, for any non-negative measurable function $f$ on $[0, \infty)$,

$$
G^{(S)} f(s)=\mathbb{E}\left[\int_{0}^{\infty} f\left(s+S_{t}\right) d t\right]=\int_{0}^{\infty} f(s+r) G^{(S)}(r) d r, \quad s \geq 0 .
$$


Since $S$ is transient, $G^{(S)} \mathbf{1}_{[0, T]}(0)<\infty$ for every $T>0$; see [35, Theorem 35.4(v)]. In fact, by [7, Proposition III.1],

$$
G^{(S)} \mathbf{1}_{[0, T]}(0) \simeq 1 / \phi(1 / T) \quad \text { on }(0, \infty)
$$

Lemma 2.2 Let

$$
w * G^{(S)}(t):=\int_{0}^{t} w(s) G^{(S)}(t-s) d s .
$$

Then $w * G^{(S)}(t) \leq 1$ for all $t>0$, and $w * G^{(S)}(t)=1$ for a.e. $t>0$.

Proof. Let $\mathcal{N} \subset(0, \infty)$ be the Borel set in Lemma 2.1. Then, for every $t \in(0, \infty) \backslash \mathcal{N}$,

$$
\begin{aligned}
w * G^{(S)}(t) & =\int_{0}^{t} w(s) G^{(S)}(t-s) d s=\int_{0}^{t} w(t-s) G^{(S)}(s) d s \\
& =\int_{0}^{\infty} \int_{0}^{t} w(t-s) \bar{p}(r, s) d s d r=\mathbb{P}\left(E_{t}<\infty\right)=1 .
\end{aligned}
$$

Furthermore, for each $t>0$, we take $t_{n} \notin \mathcal{N}$ such that $t_{n} \downarrow t$ as $n \rightarrow \infty$. Then by Fatou's lemma and the right continuity of $w$, we conclude that

$$
1=\lim _{n \rightarrow \infty} w * G^{(S)}\left(t_{n}\right) \geq \int_{0}^{\infty} \liminf _{n \rightarrow \infty} \mathbf{1}_{\left\{s \leq t_{n}\right\}} w\left(t_{n}-s\right) G^{(S)}(s) d s=\int_{0}^{t} w(t-s) G^{(S)}(s) d s .
$$

The proof is complete.

The next proposition is a key step toward the existence and uniqueness of strong solutions for general time fractional Poisson equations.

Proposition 2.3 Suppose that $\left\{P_{t}^{0} ; t \geq 0\right\}$ is a uniformly bounded and strongly continuous semigroup in some Banach space $(\mathbb{B},\|\cdot\|)$ and $T_{0} \in(0, \infty)$. Then the following hold.

(i) For every $f(t, \cdot)$ with $\int_{0}^{T_{0}}\|f(t, \cdot)\| d t<\infty$,

$$
u(t, x):=\int_{s=0}^{t} \int_{r=0}^{\infty} P_{r}^{0} f(s, \cdot)(x) \bar{p}(r, t-s) d r d s
$$

is well defined for a.e. $t \in\left[0, T_{0}\right]$ as an element in $\mathbb{B}$ such that $\int_{0}^{T_{0}}\|u(t, x)\| d t<\infty$. Moreover, for every $T \in\left(0, T_{0}\right]$,

$$
\int_{0}^{T} w(T-t) u(t, x) d t=\int_{0}^{T} \mathbb{E}\left[P_{E_{T-s}}^{0} f(s, \cdot)(x)\right] d s
$$

as elements in $\mathbb{B}$ such that

$$
\int_{0}^{T} w(T-t)\|u(t, \cdot)\| d t \leq M \int_{0}^{T}\|f(s, \cdot)\| d s,
$$

where $M:=\sup _{t>0}\left\|P_{t}^{0}\right\|<\infty$. 
(ii) If there is some constant $K \in(0, \infty)$ so that $\|f(s, \cdot)\| \leq K$ for a.e. $s \in\left(0, T_{0}\right]$, then $u(t, \cdot)$ is well defined as an element in $\mathbb{B}$ for every $t \in\left(0, T_{0}\right]$ such that $\sup _{t \in\left(0, T_{0}\right]}\|u(t, \cdot)\|<\infty$, and $t \mapsto u(t, x)$ is continuous in $(\mathbb{B},\|\cdot\|)$ with $u(t, \cdot) \rightarrow 0$ in $\mathbb{B}$ as $t \rightarrow 0$.

(iii) If $f(t, \cdot) \in \mathcal{D}(\mathcal{L})$ for a.e. $t \in\left(0, T_{0}\right]$ with $\int_{0}^{T_{0}}(\|f(t, \cdot)\|+\|\mathcal{L} f(t, \cdot)\|) d t<\infty$, then $u(t, \cdot) \in$ $\mathcal{D}(\mathcal{L})$ for a.e. $t \in\left(0, T_{0}\right]$ with $\int_{0}^{T_{0}}\|\mathcal{L} u(t, \cdot)\| d t<\infty$. Moreover, if there is some constant $K \in(0, \infty)$ so that $\|f(s, \cdot)\|+\|\mathcal{L} f(s, \cdot)\| \leq K$ for a.e. $s \in\left(0, T_{0}\right]$, then $t \mapsto \mathcal{L} u(t, x)$ is continuous in $(\mathbb{B},\|\cdot\|)$.

Proof. Throughout the proof, let $M=\sup _{t>0}\left\|P_{t}^{0}\right\|<\infty$ and $T_{0} \in(0, \infty)$.

(i) For given $f(t, \cdot)$ with $\int_{0}^{T_{0}}\|f(t, \cdot)\| d t<\infty$, by the Fubini theorem,

$$
\begin{aligned}
\int_{t=0}^{T_{0}} \int_{s=0}^{t} \int_{r=0}^{\infty}\left\|P_{r}^{0} f(s, \cdot)\right\| \bar{p}(r, t-s) d r d s d t & \leq M \int_{s=0}^{T_{0}} \int_{t=s}^{T_{0}} \int_{r=0}^{\infty}\|f(s, \cdot)\| \bar{p}(r, t-s) d r d t d s \\
& =M \int_{s=0}^{T_{0}} \int_{r=0}^{\infty}\|f(s, \cdot)\| \mathbb{P}\left(S_{r} \leq T_{0}-s\right) d r d s \\
& \leq M \int_{0}^{T_{0}}\|f(s, \cdot)\| d s G^{(S)} \mathbf{1}_{\left[0, T_{0}\right]}(0) .
\end{aligned}
$$

This establishes the well-definedness of $u(t, x)$ for a.e. $t \in\left(0, T_{0}\right]$ with

$$
\int_{0}^{T_{0}}\|u(t, \cdot)\| d t \leq M \int_{0}^{T_{0}}\|f(s, \cdot)\| d s G^{(S)} \mathbf{1}_{\left[0, T_{0}\right]}(0)<\infty .
$$

We now show that both sides of (2.5) are well defined as an element in $\mathbb{B}$. Note that, for every $T \in\left(0, T_{0}\right]$,

$$
\int_{0}^{T} \mathbb{E}\left\|P_{E_{T-s}}^{0} f(s, \cdot)\right\| d s \leq M \int_{0}^{T}\|f(s, \cdot)\| d s<\infty .
$$

This shows that $\int_{0}^{T} \mathbb{E}\left[P_{E_{T-s}}^{0} f(s, \cdot)\right] d s$ is absolutely integrable and thus it is well defined as an element in $\mathbb{B}$. Similarly, by the definition of $u(t, x)$ and the Fubini theorem,

$$
\begin{aligned}
\int_{0}^{T} w(T-t)\|u(t, \cdot)\| d t & \leq \int_{t=0}^{T} w(T-t)\left(\int_{s=0}^{t} \int_{r=0}^{\infty}\left\|P_{r}^{0} f(s, \cdot)\right\| \bar{p}(r, t-s) d r d s\right) d t \\
& \leq M \int_{s=0}^{T}\left(\int_{t=s}^{T} \int_{r=0}^{\infty} w(T-t) \bar{p}(r, t-s) d r d t\right)\|f(s, \cdot)\| d s \\
& =M \int_{s=0}^{T}\left(\int_{t=s}^{T} w(T-t) G^{(S)}(t-s) d t\right)\|f(s, \cdot)\| d s \\
& =M \int_{0}^{T}\|f(s, \cdot)\| d s<\infty
\end{aligned}
$$

where in the last equality we used Lemma 2.2. This shows that $\int_{0}^{T} w(T-t) u(t, \cdot) d t$ is absolutely integrable and thus it is well defined as an element in $\mathbb{B}$.

Next, we verify that the identity (2.5) holds. By (2.1), Lemma 2.2 and the Fubini theorem,

$$
\int_{0}^{T} \mathbb{E}\left[P_{E_{T-s}}^{0} f(s, \cdot)(x)\right] d s=\int_{s=0}^{T} \int_{r=0}^{\infty} P_{r}^{0} f(s, \cdot)(x) d_{r} \mathbb{P}\left(E_{T-s} \leq r\right) d s
$$




$$
\begin{aligned}
& =\int_{s=0}^{T} \int_{r=0}^{\infty} P_{r}^{0} f(s, \cdot)(x)\left(\int_{t=0}^{T-s} w(T-s-t) \bar{p}(r, t) d t\right) d r d s \\
& =\int_{s=0}^{T} \int_{r=0}^{\infty} P_{r}^{0} f(s, \cdot)(x)\left(\int_{t=s}^{T} w(T-t) \bar{p}(r, t-s) d t\right) d r d s \\
& =\int_{t=0}^{T}\left(\int_{s=0}^{t} \int_{r=0}^{\infty} P_{r}^{0} f(s, \cdot)(x) \bar{p}(r, t-s) d r d s\right) w(T-t) d t \\
& =\int_{0}^{T} w(T-t) u(t, x) d t .
\end{aligned}
$$

This establishes (2.5).

(ii) If $\|f(s, \cdot)\| \leq K<\infty$ for a.e. $s \in\left(0, T_{0}\right]$, then, by (2.3) we have that for every $t \in\left(0, T_{0}\right]$,

$$
\begin{aligned}
\int_{s=0}^{t} \int_{r=0}^{\infty}\left\|P_{r}^{0} f(s, \cdot)\right\| \bar{p}(r, t-s) d r d s & \leq M \int_{s=0}^{t} \int_{r=0}^{\infty}\|f(s, \cdot)\| \bar{p}(r, t-s) d r d s \\
& \leq M \int_{0}^{t}\|f(s, \cdot)\| G^{(S)}(t-s) d s \\
& \leq M K G^{(S)} \mathbf{1}_{[0, t]}(0) \leq M K \frac{c_{0}}{\phi(1 / t)}
\end{aligned}
$$

So $u(t, x):=\int_{s=0}^{t} \int_{r=0}^{\infty} P_{r}^{0} f(s, \cdot) \bar{p}(r, t-s) d r d s$ is well defined as an element in $\mathbb{B}$ and that for every $t \in\left(0, T_{0}\right], \sup _{t \in\left(0, T_{0}\right]}\|u(t, \cdot)\| \leq M K c_{0} / \phi\left(1 / T_{0}\right)<\infty$. Since $\lim _{r \uparrow \infty} \phi(r)=\nu(0, \infty)=\infty$, we have $\lim _{t \downarrow 0}\|u(t, \cdot)\|=0$.

We now show the continuity of $t \mapsto u(t, x)$ in $(\mathbb{B},\|\cdot\|)$. Indeed, for any $0<t_{1} \leq t_{2}$,

$$
\begin{aligned}
u\left(t_{2}, x\right)-u\left(t_{1}, x\right)= & \int_{s=t_{1}}^{t_{2}} \int_{r=0}^{\infty} P_{r}^{0} f(s, \cdot)(x) \bar{p}\left(r, t_{2}-s\right) d r d s \\
& +\int_{s=0}^{t_{1}} \int_{r=0}^{\infty} P_{r}^{0} f(s, \cdot)(x)\left(\bar{p}\left(r, t_{2}-s\right)-\bar{p}\left(r, t_{1}-s\right)\right) d r d s .
\end{aligned}
$$

Thus,

$$
\begin{aligned}
\left\|u\left(t_{2}, \cdot\right)-u\left(t_{1}, \cdot\right)\right\| \leq & \int_{s=t_{1}}^{t_{2}} \int_{r=0}^{\infty}\left\|P_{r}^{0} f(s, \cdot)\right\| \bar{p}\left(r, t_{2}-s\right) d r d s \\
& +\int_{s=0}^{t_{1}} \int_{r=0}^{\infty}\left\|P_{r}^{0} f(s, \cdot)\right\|\left|\bar{p}\left(r, t_{2}-s\right)-\bar{p}\left(r, t_{1}-s\right)\right| d r d s \\
\leq & M K \int_{s=t_{1}}^{t_{2}} \int_{r=0}^{\infty} \bar{p}\left(r, t_{2}-s\right) d r d s \\
& +M K \int_{s=0}^{t_{1}} \int_{r=0}^{\infty}\left|\bar{p}\left(r, t_{2}-s\right)-\bar{p}\left(r, t_{1}-s\right)\right| d r d s \\
= & M K G^{(S)} \mathbf{1}_{\left[0, t_{2}-t_{1}\right]}(0)+M K \int_{s=0}^{t_{1}} \int_{r=0}^{\infty}\left|\bar{p}\left(r, t_{2}-t_{1}+s\right)-\bar{p}(r, s)\right| d r d s .
\end{aligned}
$$

Using (2.3) and the fact $\lim _{r \uparrow \infty} \phi(r)=\nu(0, \infty)=\infty$ in the first term, and the $L^{1}$-continuity of the translation of the integrable function $(r, s) \mapsto \bar{p}(r, s)$ on $(0, \infty) \times\left(0, T_{0}\right]$ on the second term, we see that both terms go to zero as $t_{2}-t_{1} \rightarrow 0$. Thus the desired assertion follows. 
(iii) Suppose that $f(t, \cdot) \in \mathcal{D}(\mathcal{L})$ for a.e. $t \in\left(0, T_{0}\right]$ with $\int_{0}^{T_{0}}(\|f(t, \cdot)\|+\|\mathcal{L} f(t, \cdot)\|) d t<\infty$. Note that for a.e. $s \in\left[0, T_{0}\right]$ and every $r>0, \mathcal{L} P_{r}^{0} f(s, \cdot)=P_{r}^{0} \mathcal{L} f(s, \cdot)$ and $\left\|\mathcal{L} P_{r}^{0} f(s, \cdot)\right\|=\left\|P_{r}^{0} \mathcal{L} f(s, \cdot)\right\| \leq$ $M\|\mathcal{L} f(s, \cdot)\|$. By the similar calculation as that in (2.7), we have

$$
\int_{t=0}^{T_{0}} \int_{s=0}^{T_{0}} \int_{r=0}^{\infty}\left\|\mathcal{L} P_{r}^{0} f(s, \cdot)\right\| \bar{p}(r, t-s) d r d s d t \leq M \int_{0}^{T_{0}}\|\mathcal{L} f(s, \cdot)\| d s G^{(S)} \mathbf{1}_{\left[0, T_{0}\right]}(0)<\infty .
$$

Thus, by (2.4) and the closed graph theorem, $u(t, \cdot) \in \mathcal{D}(\mathcal{L})$ for a.e. $t \in\left[0, T_{0}\right]$ such that

$$
\int_{0}^{T_{0}}\|\mathcal{L} u(s, \cdot)\| d s \leq c \int_{0}^{T_{0}}\|\mathcal{L} f(s, \cdot)\| d s G^{(S)} \mathbf{1}_{\left[0, T_{0}\right]}(0)<\infty .
$$

Next, we suppose that $\|f(s, \cdot)\|+\|\mathcal{L} f(s, \cdot)\| \leq K<\infty$ for a.e. $s \in\left(0, T_{0}\right]$. Then, by the closed graph theorem, the Riemann sum approximation and the fact that $\mathcal{L} P_{r}^{0} f(s, \cdot)=P_{r}^{0} \mathcal{L} f(s, \cdot)$ for a.e. $s \in\left(0, T_{0}\right]$ and any $r>0$, we have that for all $0 \leq T \leq T_{0}$.

$$
\int_{0}^{T} \mathcal{L} u(t, \cdot)(x) d t=\int_{t=0}^{T} \int_{s=0}^{t} \int_{r=0}^{\infty} P_{r}^{0} \mathcal{L} f(s, \cdot)(x) \bar{p}(r, t-s) d r d s d t .
$$

Using (2.12), we follow exactly the same arguments these in (2.9) and (2.10) and get that for any $0<t_{1} \leq t_{2}$,

$$
\begin{aligned}
\left\|\mathcal{L} u\left(t_{2}, \cdot\right)-\mathcal{L} u\left(t_{1}, \cdot\right)\right\| \leq & \int_{s=t_{1}}^{t_{2}} \int_{r=0}^{\infty}\left\|P_{r}^{0} \mathcal{L} f(s, \cdot)\right\| \bar{p}\left(r, t_{2}-s\right) d r d s \\
& +\int_{s=0}^{t_{1}} \int_{r=0}^{\infty}\left\|P_{r}^{0} \mathcal{L} f(s, \cdot)\right\|\left|\bar{p}\left(r, t_{2}-s\right)-\bar{p}\left(r, t_{1}-s\right)\right| d r d s \\
\leq & M K G^{(S)} \mathbf{1}_{\left[0, t_{2}-t_{1}\right]}(0)+M K \int_{s=0}^{t_{1}} \int_{r=0}^{\infty}\left|\bar{p}\left(r, t_{2}-t_{1}+s\right)-\bar{p}(r, s)\right| d r d s .
\end{aligned}
$$

Therefore, $t \mapsto \mathcal{L} u(t, x)$ is continuous in $(\mathbb{B},\|\cdot\|)$. This completes the proof of the proposition.

Remark 2.4 If we assume

$$
\int_{0}^{T_{0}}\|f(s, \cdot)\| G^{(S)}(t-s) d s<\infty \text { with } \lim _{t \rightarrow 0} \int_{0}^{t}\|f(s, \cdot)\| G^{(S)}(t-s) d s=0
$$

in place of $\|f(t, \cdot)\| \leq K$ for a.e. $t \in\left(0, T_{0}\right]$ in Proposition $2.3($ ii), then, by the same proof, $u(t, \cdot)$ is well defined as an element in $\mathbb{B}$ for every $t \in\left(0, T_{0}\right]$ with $u(t, \cdot) \rightarrow 0$ in $\mathbb{B}$ as $t \rightarrow 0$.

Recall from [8, (2.3)] that

$$
\int_{0}^{\infty} e^{-\lambda x} w(x) d x=\frac{\phi(\lambda)}{\lambda} \text { for } \lambda>0
$$

Proof of Theorem 1.1. (a) (Existence) The case that $f \equiv 0$ is proved in [8, Theorem 2.3]. Thus by the linearity we only need to show the existence of the solution when $g \equiv 0$, which we will assume throughout the proof. 
Let $u(t, x)$ be defined by (2.4). Part (i) has been proved in Proposition 2.3(ii) as $\|f(t, \cdot)\| \leq K$ for a.e. $t \in\left(0, T_{0}\right]$, while part (ii) has been established in Proposition [2.3)(iii).

By (2.11), using the closed graph theorem and the Riemann sum approximation, we have that for every $T \in\left(0, T_{0}\right]$,

$$
\int_{0}^{T} \mathcal{L} u(t, \cdot)(x) d t=\int_{t=0}^{T} \int_{s=0}^{t} \int_{r=0}^{\infty} \mathcal{L} P_{r}^{0} f(s, \cdot)(x) \bar{p}(r, t-s) d r d s d t .
$$

Thus, for every $T \in\left(0, T_{0}\right]$, using the Fubini theorem and the integration by parts,

$$
\begin{aligned}
\int_{0}^{T} \mathcal{L} u(t, \cdot)(x) d t & =\int_{s=0}^{T} \int_{r=0}^{\infty} \mathcal{L} P_{r}^{0} f(s, \cdot)(x)\left(\int_{t=s}^{T} \bar{p}(r, t-s) d t\right) d r d s \\
& =\int_{s=0}^{T}\left(\int_{r=0}^{\infty} \frac{d}{d r} P_{r}^{0} f(s, \cdot)(x) \mathbb{P}\left(S_{r} \leq T-s\right) d r\right) d s \\
& =\int_{s=0}^{T}\left(\left.P_{r}^{0} f(s, \cdot)(x) \mathbb{P}\left(S_{r} \leq T-s\right)\right|_{r=0} ^{\infty}-\int_{r=0}^{\infty} P_{r}^{0} f(s, \cdot)(x) d_{r} \mathbb{P}\left(S_{r} \leq T-s\right)\right) d s \\
& =\int_{s=0}^{T}\left(-f(s, x)+\int_{r=0}^{\infty} P_{r}^{0} f(s, \cdot)(x) d_{r} \mathbb{P}\left(E_{T-s} \leq r\right)\right) d s .
\end{aligned}
$$

Hence,

$$
\int_{0}^{T}(f(t, x)+\mathcal{L} u(t, \cdot)(x)) d t=\int_{0}^{T} \mathbb{E}\left[P_{E_{T-s}}^{0} f(s, \cdot)(x)\right] d s=\int_{0}^{T} w(T-t) u(t, x) d t,
$$

where the last equality is due to (2.5). The proof of (iii) is finished.

(b) (Uniqueness) Suppose that $u$ and $v$ are two (strong) solutions of $\partial_{t}^{w} u(t, x)=\mathcal{L} u(t, x)+$ $f(t, x)$ on $\left(0, T_{0}\right] \times E$ with $u(0, x)=g(x)$. Then $h:=u-v$ is a (strong) solution of

$$
\partial_{t}^{w} h(t, x)=\mathcal{L} h(t, x) \quad \text { on }\left(0, T_{0}\right] \times E \text { with } h(0, \cdot)=0 .
$$

By the definition of the strong solution, we know that $t \mapsto h(t, \cdot)$ is continuous on $\left[0, T_{0}\right]$ with $\lim _{t \rightarrow 0} h(t, \cdot)=0$ and $K_{1}:=\sup _{t \in\left[0, T_{0}\right]}\|h(t, \cdot)\|<\infty ;$ moreover,

$$
h(t, \cdot) \in \mathcal{D}(\mathcal{L}) \text { for a.e. } t \in\left(0, T_{0}\right] \text { with } \int_{0}^{T_{0}}\|\mathcal{L} h(t, \cdot)\| d t<\infty .
$$

Define

$$
\bar{f}(t, x):=\int_{\left(t, T_{0}+t\right]} h\left(T_{0}+t-s, x\right) \nu(d s), \quad t \geq 0 .
$$

Clearly, $\|\bar{f}(t, \cdot)\| \leq K_{1} w(t)$ for $t>0$, and so by (2.13) we have that for every $\lambda>0$,

$$
\int_{0}^{\infty} e^{-\lambda t}\|\bar{f}(t, \cdot)\| d t \leq K_{1} \int_{0}^{\infty} e^{-\lambda t} w(t) d t=K_{1} \phi(\lambda) / \lambda .
$$

Moreover, since $\int_{0}^{\infty} e^{-\lambda t} \nu(d t)=\phi^{\prime}(\lambda) \lambda \leq \phi(\lambda)$, by (2.16) and the Fubini theorem, we have that 
for every $\lambda>0$

$$
\begin{aligned}
\int_{0}^{\infty} e^{-\lambda t} \int_{t}^{T_{0}+t}\left\|\mathcal{L} h\left(T_{0}+t-s, \cdot\right)\right\| \nu(d s) d t & =\int_{0}^{\infty} \int_{\left(s-T_{0}\right)^{+}}^{s} e^{-\lambda t}\left\|\mathcal{L} h\left(T_{0}+t-s, \cdot\right)\right\| d t \nu(d s) \\
& =\int_{0}^{\infty} \int_{\left(T_{0}-s\right)^{+}}^{T_{0}} e^{-\lambda\left(r+s-T_{0}\right)}\|\mathcal{L} h(r, \cdot)\| d r \nu(d s) \\
& \leq e^{\lambda T_{0}}\left(\int_{0}^{\infty} e^{-\lambda s} \nu(d s)\right)\left(\int_{0}^{T_{0}}\|\mathcal{L} h(r, \cdot)\| d r\right) \\
& \leq e^{\lambda T_{0}} \phi(\lambda) \int_{0}^{T_{0}}\|\mathcal{L} h(t, \cdot)\| d t<\infty .
\end{aligned}
$$

Hence, by the closed graph theorem and the Riemann sum approximation, we conclude that $\bar{f}(t, \cdot) \in$ $\mathcal{D}(\mathcal{L})$ for a.e. $t>0$, and

$$
\mathcal{L} \bar{f}(t, \cdot)(x)=\int_{\left(t, T_{0}+t\right]} \mathcal{L} h\left(T_{0}+t-s, \cdot\right)(x) \nu(d s) \quad \text { for a.e. } t>0
$$

with

$$
\int_{0}^{\infty} e^{-\lambda t}\|\mathcal{L} \bar{f}(t, \cdot)\| d t \leq e^{\lambda T_{0}} \phi(\lambda) \int_{0}^{T_{0}}\|\mathcal{L} h(t, \cdot)\| d t<\infty
$$

Let

$$
\bar{h}(t, x):=\int_{s=0}^{t} \int_{r=0}^{\infty} P_{r}^{0} \bar{f}(s, \cdot)(x) \bar{p}(r, t-s) d r d s, \quad t>0,
$$

and $M:=\sup _{t>0}\left\|P_{t}^{0}\right\|<\infty$. By (2.18), (2.20) and Proposition 2.3, for a.e. $t \in[0, \infty), \bar{h}(t, \cdot)$ is well defined as an element in $\mathcal{D}(\mathcal{L}) \subset \mathbb{B}$ with $\int_{0}^{T}(\|\mathcal{L} \bar{h}(t, \cdot)\|+\|\bar{h}(t, \cdot)\|) d t<\infty$ for every $T>0$. Moreover, by (2.18) and the Fubini theorem, for every $\lambda>0$

$$
\begin{aligned}
\int_{0}^{\infty} e^{-\lambda t}\|\bar{h}(t, \cdot)\| d t & \leq \int_{t=0}^{\infty} e^{-\lambda t} \int_{s=0}^{t} \int_{r=0}^{\infty}\left\|P_{r}^{0} \bar{f}(s, \cdot)\right\| \bar{p}(r, t-s) d r d s d t \\
& \leq M \int_{s=0}^{\infty} \int_{t=s}^{\infty} e^{-\lambda t} \int_{r=0}^{\infty}\|\bar{f}(s, \cdot)\| \bar{p}(r, t-s) d r d t d s \\
& =M \int_{s=0}^{\infty} e^{-\lambda s}\|\bar{f}(s, \cdot)\|\left(\int_{t=s}^{\infty} e^{-\lambda(t-s)} \int_{r=0}^{\infty} \bar{p}(r, t-s) d r d t\right) d s \\
& =\frac{M}{\phi(\lambda)} \int_{s=0}^{\infty} e^{-\lambda s}\|\bar{f}(s, \cdot)\| d s \leq M K_{1} / \lambda<\infty
\end{aligned}
$$

Here in the last equality we used the fact that

$$
\int_{t=s}^{\infty} e^{-\lambda(t-s)} \int_{r=0}^{\infty} \bar{p}(r, t-s) d r d t=\int_{r=0}^{\infty} \mathbb{E} e^{-\lambda S_{r}} d r=\int_{0}^{\infty} e^{-r \phi(\lambda)} d r=1 / \phi(\lambda),
$$

and the last inequality follows from (2.18). In view of all the estimates above, we have by the same argument as that for (2.14) (where the boundedness of $t \mapsto\|f(t, \cdot)\|$ is not needed) that for every $T>0$,

$$
\int_{0}^{T}(\bar{f}(t, x)+\mathcal{L} \bar{h}(t, \cdot)(x)) d t=\int_{0}^{T} w(T-t) \bar{h}(t, x) d t
$$


Now, we extend $h(t, x)$ on $(0, \infty) \times E$ by defining $h(t, x)=\bar{h}\left(t-T_{0}, x\right)$ for $t>T_{0}$. Then, since for all $T>T_{0}$

$$
\begin{aligned}
\int_{0}^{T-T_{0}} \bar{f}(t, x) d t & =\int_{0}^{T-T_{0}} \int_{\left(t, T_{0}+t\right]} h\left(T_{0}+t-s, x\right) \nu(d s) d t \\
& =\int_{(0, T]} \int_{0 \vee\left(s-T_{0}\right)}^{s \wedge\left(T-T_{0}\right)} h\left(T_{0}+t-s, x\right) d t \nu(d s) \\
& =\int_{(0, T]} \int_{0 \vee\left(T_{0}-s\right)}^{T_{0} \wedge(T-s)} h(t, x) d t \nu(d s) \\
& =\int_{0}^{T_{0}}\left(\int_{\left(T_{0}-t, T-t\right]} \nu(d s)\right) h(t, x) d t \\
& =\int_{0}^{T_{0}}\left(w\left(T_{0}-t\right)-w(T-t)\right) h(t, x) d t
\end{aligned}
$$

we have that, by (2.15), (2.25) and (2.24), for all $T>T_{0}$

$$
\begin{aligned}
\int_{0}^{T} \mathcal{L} h(t, \cdot)(x) d t= & \int_{0}^{T_{0}} w\left(T_{0}-t\right) h(t, x) d t+\int_{T_{0}}^{T} \mathcal{L} \bar{h}\left(t-T_{0}, x\right) d t \\
= & \int_{0}^{T_{0}} w(T-t) h(t, x) d t+\int_{0}^{T_{0}}\left(w\left(T_{0}-t\right)-w(T-t)\right) h(t, x) d t \\
& +\int_{0}^{T-T_{0}} \mathcal{L} \bar{h}(t, x) d t \\
= & \int_{0}^{T_{0}} w(T-t) h(t, x) d t+\int_{0}^{T-T_{0}}(\bar{f}(t, x) d t+\mathcal{L} \bar{h}(t, x)) d t \\
= & \int_{0}^{T_{0}} w(T-t) h(t, x) d t+\int_{0}^{T-T_{0}} w\left(T-T_{0}-t\right) \bar{h}(t, x) d t \\
= & \int_{0}^{T_{0}} w(T-t) h(t, x) d t+\int_{T_{0}}^{T} w(T-t) \bar{h}\left(t-T_{0}, x\right) d t \\
= & \int_{0}^{T} w(T-t) h(t, x) d t .
\end{aligned}
$$

This shows that $h$ solves

$$
\partial_{t}^{w} h(t, x)=\mathcal{L} h(t, x) \quad \text { for all } t>0 \text { with } h(0, x)=0
$$


To show that $h(t, \cdot)=0$ to a.e. $t>0$, first note that for every $\lambda>0$,

$$
\begin{aligned}
& \int_{0}^{\infty} e^{-\lambda t}\|\mathcal{L} h(t, \cdot)\| d t \\
& =\int_{0}^{T_{0}} e^{-\lambda t}\|\mathcal{L} h(t, \cdot)\| d t+\int_{T_{0}}^{\infty} e^{-\lambda t}\left\|\mathcal{L} \bar{h}\left(t-T_{0}, \cdot\right)\right\| d t \\
& \leq \int_{0}^{T_{0}}\|\mathcal{L} h(t, \cdot)\| d t+\int_{0}^{\infty} e^{-\lambda t}\|\mathcal{L} \bar{h}(t, \cdot)\| d t \\
& \leq \int_{0}^{T_{0}}\|\mathcal{L} h(t, \cdot)\| d t+\int_{t=0}^{\infty} e^{-\lambda t} \int_{s=0}^{t} \int_{r=0}^{\infty}\left\|\mathcal{L} P_{r}^{0} \bar{f}(s, \cdot)\right\| \bar{p}(r, t-s) d r d s d t \\
& \leq \int_{0}^{T_{0}}\|\mathcal{L} h(t, \cdot)\| d t+M \int_{s=0}^{\infty} \int_{t=s}^{\infty} e^{-\lambda t} \int_{r=0}^{\infty}\|\mathcal{L} \bar{f}(s, \cdot)\| \bar{p}(r, t-s) d r d t d s \\
& =\int_{0}^{T_{0}}\|\mathcal{L} h(t, \cdot)\| d t+M \int_{s=0}^{\infty} e^{-\lambda s}\|\mathcal{L} \bar{f}(s, \cdot)\|\left(\int_{t=s}^{\infty} e^{-\lambda(t-s)} \int_{r=0}^{\infty} \bar{p}(r, t-s) d r d t\right) d s \\
& =\int_{0}^{T_{0}}\|\mathcal{L} h(t, \cdot)\| d t+\frac{M}{\phi(\lambda)} \int_{s=0}^{\infty} e^{-\lambda s}\|\mathcal{L} \bar{f}(s, \cdot)\| d s \\
& \leq\left(1+M e^{\lambda T_{0}}\right) \int_{0}^{T_{0}}\|\mathcal{L} h(t, \cdot)\| d t<\infty,
\end{aligned}
$$

where (2.23) was used the last equality, and (2.16) and (2.20) were used in the last inequality. Now repeating the proof of the uniqueness part in [8, Theorem 2.3], we can show that $h(t, \cdot)=0$ for a.e. $t>0$. Indeed, let $H(\lambda, x):=\int_{0}^{\infty} e^{-\lambda t} h(t, x) d t, \lambda>0$, be the Laplace transform of $t \mapsto h(t, x)$. By (2.22) for every $\lambda>0, H(\lambda, \cdot) \in \mathbb{B}$ with $\|H(\lambda, \cdot)\|<\infty$. By the closed graph theorem, the Riemann sum approximation and (2.27), for each $\lambda>0, H(\lambda, \cdot) \in \mathcal{D}(\mathcal{L})$ with

$$
\mathcal{L} H(\lambda, \cdot)=\int_{0}^{\infty} e^{-\lambda t} \mathcal{L} h(t, \cdot) d t \quad \text { and } \quad\|\mathcal{L} H(\lambda, \cdot)\| \leq \int_{0}^{\infty} e^{-\lambda t}\|\mathcal{L} h(t, \cdot)\| d t<\infty .
$$

Taking the Laplace transform in $t$ on both sides of (2.26) yields

$$
H(\lambda, x) \frac{\phi(\lambda)}{\lambda}=H(\lambda, x) \int_{0}^{\infty} e^{-\lambda s} w(s) d s=\frac{1}{\lambda} \int_{0}^{\infty} e^{-\lambda t} \mathcal{L} h(t, x) d t=\frac{\mathcal{L} H(\lambda, x)}{\lambda} .
$$

Thus $(\phi(\lambda)-\mathcal{L}) H(\lambda, x)=0$ for every $\lambda>0$. Since $\mathcal{L}$ is the infinitesimal generator of a uniformly bounded and strongly continuous semigroup $\left\{P_{t}^{0}, t \geq 0\right\}$ in Banach space $\mathbb{B}$, for every $\alpha>0$, the resolvent $G_{\alpha}^{0}=\int_{0}^{\infty} e^{-\alpha t} P_{t}^{0} d t$ is well defined and is the inverse to $\alpha-\mathcal{L}$. Hence $H(\lambda, \cdot)=0$ in $\mathbb{B}$ for every $\lambda>0$. By the uniqueness of the Laplace transform, we conclude that $h(t, \cdot)=0$ for a.e. $t>0$.

Since $t \mapsto h(t, \cdot)$ is continuous in $\mathbb{B}$ for $t \in\left[0, T_{0}\right]$, we conclude that $h(t, \cdot)=0$ in $\mathbb{B}$ for every $t \in\left[0, T_{0}\right]$. This establishes the uniqueness.

Remark 2.5 If we assume that there is some positive constant $K$ so that $\|\mathcal{L} f(t, \cdot)\| \leq K$ for a.e. $t \in\left(0, T_{0}\right]$ instead of $\int_{0}^{T_{0}}\|\mathcal{L} f(t, \cdot)\| d t<\infty$ in Theorem 1.1, then its conclusion (ii) can be strengthened to

(ii') $u(t, \cdot) \in \mathcal{D}(\mathcal{L})$ for every $t \in\left(0, T_{0}\right]$ and $\sup _{t \in\left[0, T_{0}\right]}\|\mathcal{L} u(t, \cdot)\|<\infty$. 
Its proof is similar to the last part of that for Proposition 2.3.

To study weak solutions for general time fractional Poisson equations, we will assume that $(\mathcal{L}, \mathcal{D}(\mathcal{L}))$ is the infinitesimal generator of a (symmetric) Dirichlet form on $L^{2}(E ; \mu)$. Its associated transition semigroup is denoted as $\left\{P_{t}^{0} ; t \geq 0\right\}$, which is a strongly continuous contraction semigroup in $L^{2}(E ; \mu)$. Denote by $\langle\cdot, \cdot\rangle$ the inner product of $L^{2}(E ; \mu)$.

Proposition 2.6 Suppose that $f(t, x)$ is a function on $\left(0, T_{0}\right] \times E$ with $\int_{0}^{T_{0}}\|f(t, \cdot)\|_{L^{2}(E ; \mu)} d t<\infty$ for some $T_{0} \in(0, \infty)$. Then the following hold.

(1) $u(t, x):=\int_{s=0}^{t} \int_{r=0}^{\infty} P_{r}^{0} f(s, \cdot) \bar{p}(r, t-s) d r d s$ is well defined as an element in $L^{2}(E ; \mu)$ for a.e. $t \in\left(0, T_{0}\right]$, and it is a weak solution of $\partial_{t}^{w} u=\mathcal{L} u+f(t, x)$ on $\left(0, T_{0}\right] \times E$ with $u(0, x)=0$ in the following sense:

(i) $u(t, \cdot) \in L^{2}(E ; \mu)$ for a.e. $t \in\left(0, T_{0}\right]$ with $\int_{0}^{T_{0}}\|u(t, \cdot)\|_{L^{2}(E ; \mu)} d t<\infty$. Moreover, for every $T \in\left(0, T_{0}\right]$

$$
\int_{0}^{T} w(T-t)\|u(t, \cdot)\|_{L^{2}(E ; \mu)} d t<\infty
$$

and so $\int_{0}^{T} w(T-t) u(t, \cdot) d t$ is well defined as an element in $L^{2}(E ; m)$.

(ii) For every $\varphi \in \mathcal{D}(\mathcal{L})$ and $T \in\left(0, T_{0}\right]$,

$$
\left\langle\int_{0}^{T} w(T-s) u(s, \cdot) d s, \varphi\right\rangle=\int_{0}^{T}\langle f(s, \cdot), \varphi\rangle d s+\int_{0}^{T}\langle u(s, \cdot), \mathcal{L} \varphi\rangle d s
$$

(2) If we further assume that

$$
\|f(t, \cdot)\|_{L^{2}(E ; \mu)} \leq K<\infty \text { for a.e. } t \in\left(0, T_{0}\right]
$$

then the conclusion (i) of (1) can be strengthened to

(i') $u(t, \cdot)$ is well defined as an element in $L^{2}(E ; \mu)$ for every $t \in\left(0, T_{0}\right]$ satisfying that $t \mapsto u(t, x)$ is continuous in $L^{2}(E ; \mu)$,

$$
\sup _{t \in\left(0, T_{0}\right]}\|u(t, \cdot)\|_{L^{2}(E ; \mu)}<\infty \quad \text { and } \quad u(t, \cdot) \rightarrow 0 \text { in } L^{2}(E ; \mu) \text { as } t \rightarrow 0 .
$$

If in addition, $f(t, \cdot) \in \mathcal{D}(\mathcal{L})$ for a.e. $t \in\left(0, T_{0}\right]$ with $\|\mathcal{L} f(t, \cdot)\|_{L^{2}(E ; \mu)} \leq K<\infty$ for a.e. $t \in\left(0, T_{0}\right]$, then $t \mapsto \mathcal{L} u(t, x)$ is continuous in $L^{2}(E ; \mu)$.

Proof. (1) Let $u(t, x):=\int_{s=0}^{t} \int_{r=0}^{\infty} P_{r}^{0} f(s, \cdot) \bar{p}(r, t-s) d r d s$. By Proposition 2.3(i), $u(t, x)$ is well defined for a.e. $t \in\left[0, T_{0}\right]$ as an element in $L^{2}(E ; \mu)$ having $\int_{0}^{T_{0}}\|u(t, x)\|_{L^{2}(E ; \mu)} d t<\infty$. It follows from (2.6) that for every $T \in\left(0, T_{0}\right]$,

$$
\int_{0}^{T} w(T-t)\|u(t, \cdot)\|_{L^{2}(E ; \mu)} d t \leq M \int_{0}^{T}\|f(s, \cdot)\|_{L^{2}(E ; \mu)} d s<\infty
$$

with $M=\sup _{t>0}\left\|P_{t}^{0}\right\|$. Hence $\int_{0}^{T} w(T-t) u(t, \cdot) d t$ is well defined as element in $L^{2}(E ; \mu)$. 

$P_{r}^{0}$

For every $\varphi \in \mathcal{D}(\mathcal{L})$ and $T \in\left(0, T_{0}\right]$, by (2.5), (2.30), the Fubini theorem and the symmetry of

$$
\begin{aligned}
\left\langle\int_{0}^{T} w(T-t) u(t, \cdot) d t, \varphi\right\rangle & =\left\langle\int_{0}^{T} \mathbb{E} P_{E_{T-s}}^{0} f(s, \cdot) d s, \varphi\right\rangle \\
& =\int_{0}^{T}\left\langle f(s, \cdot), \mathbb{E} P_{E_{T-s}}^{0} \varphi\right\rangle d s \\
& =\int_{0}^{T}\langle f(s, \cdot), \varphi\rangle d s+\int_{0}^{T}\left\langle f(s, \cdot), \mathbb{E} \int_{0}^{E_{T-s}} P_{t}^{0} \mathcal{L} \varphi d t\right\rangle d s \\
& =\int_{0}^{T}\langle f(s, \cdot), \varphi\rangle d s+\int_{0}^{T}\left\langle\mathbb{E} \int_{0}^{E_{T-s}} P_{t}^{0} f(s, \cdot) d t, \mathcal{L} \varphi\right\rangle d s .
\end{aligned}
$$

By the Fubini theorem,

$$
\begin{aligned}
\mathbb{E} \int_{0}^{E_{T-s}} P_{t}^{0} f(s, \cdot) d t & =\int_{t=0}^{\infty} \mathbb{P}\left(E_{T-s}>t\right) P_{t}^{0} f(s, \cdot) d t=\int_{t=0}^{\infty} \mathbb{P}\left(S_{t} \leq T-s\right) P_{t}^{0} f(s, \cdot) d t \\
& =\int_{t=0}^{\infty}\left(\int_{r=0}^{T-s} \bar{p}(t, r) d r\right) P_{t}^{0} f(s, \cdot) d t=\int_{r=0}^{T-s} \int_{t=0}^{\infty} P_{t}^{0} f(s, \cdot) \bar{p}(t, r) d t d r \\
& =\int_{r=0}^{T-s} \int_{t=0}^{\infty} P_{t}^{0} f(s, \cdot) \bar{p}(t, T-s-r) d t d r .
\end{aligned}
$$

Hence

$$
\begin{aligned}
\int_{0}^{T}\left(\mathbb{E} \int_{0}^{E_{T-s}} P_{t}^{0} f(s, \cdot) d t\right) d s & =\int_{s=0}^{T} \int_{r=0}^{T-s} \int_{t=0}^{\infty} P_{t}^{0} f(s, \cdot) \bar{p}(t, T-s-r) d t d r d s \\
& =\int_{r=0}^{T} \int_{s=0}^{T-r} \int_{t=0}^{\infty} P_{t}^{0} f(s, \cdot) \bar{p}(t, T-r-s) d t d s d r \\
& =\int_{0}^{T} u(T-r, \cdot) d r .
\end{aligned}
$$

This combined with (2.31) shows that (2.28) holds for every $\varphi \in \mathcal{D}(\mathcal{L})$. Therefore, $u(t, x)$ is a weak solution to $\partial_{t}^{w} u=\mathcal{L} u+f(t, x)$ on $\left(0, T_{0}\right] \times E$.

(2) If (2.29) holds, the conclusion (i') follows from Proposition 2.3(ii). If, in addition, $\|\mathcal{L} f(t, \cdot)\|_{L^{2}(E ; \mu)}$ $\leq K<\infty$ for a.e. $t \in\left(0, T_{0}\right]$, the continuity of $t \mapsto \mathcal{L} u(t, x)$ in $L^{2}(E ; \mu)$ follows from Proposition 2.3 (iii).

Proof of Theorem 1.3, That $u(t, \cdot)$ defined by (1.6) is a weak solution of

$$
\partial_{t}^{w} u=\mathcal{L} u+f(t, \cdot) \quad \text { on }\left(0, T_{0}\right] \times E \text { with } u(0, \cdot)=g
$$

follows directly from Proposition 2.6, [13, Theorem 2.4] and the linearity. So it remains to establish $u$ is the unique weak solution of (2.32).

Suppose $v$ is another weak solution of (2.32). Then $h:=u-v$ is a weak solution of

$$
\partial_{t}^{w} h=\mathcal{L} h \quad \text { on }\left[0, T_{0}\right] \times E \text { with } h(0, \cdot)=0 .
$$


As in the proof of the uniqueness part of Theorem 1.1, define $\bar{f}(t, x)$ and $\bar{h}(t, x)$ as (2.17) and (2.21) respectively. Since (2.18) holds in this case, by Proposition 2.6(1), $\bar{h}(t, x)$ is a weak solution to

$$
\partial_{t}^{w} \bar{h}=\mathcal{L} \bar{h}(t, x)+\bar{f}(t, x) \quad \text { on } \quad(0, \infty) \times E \quad \text { with } \quad \bar{h}(0, x)=0 .
$$

Moreover, 2.22) also holds.

Now define $h(t, x)=\bar{h}\left(t-T_{0}, x\right)$ for $t>T_{0}$. Then, by (2.25), (2.33) and (2.34), we have that for all $T>T_{0}$ and $\varphi \in \mathcal{D}(\mathcal{L})$

$$
\begin{aligned}
\int_{0}^{T}\langle\mathcal{L} \varphi, h(t, \cdot)\rangle d t= & \left\langle\int_{0}^{T_{0}} w\left(T_{0}-s\right) h(t, \cdot) d t, \varphi\right\rangle+\int_{T_{0}}^{T}\left\langle\mathcal{L} \varphi, \bar{h}\left(t-T_{0}, \cdot\right)\right\rangle d t \\
= & \left\langle\int_{0}^{T_{0}} w(T-s) h(t, \cdot) d t, \varphi\right\rangle+\left\langle\int_{0}^{T_{0}}\left(w\left(T_{0}-t\right)-w(T-t)\right) h(t, \cdot) d t, \varphi\right\rangle \\
& +\int_{0}^{T-T_{0}}\langle\mathcal{L} \varphi, \bar{h}(t, \cdot) d t\rangle \\
= & \left\langle\int_{0}^{T_{0}} w(T-s) h(t, \cdot) d t, \varphi\right\rangle+\int_{0}^{T-T_{0}}\langle\bar{f}(t, \cdot), \varphi\rangle d t+\int_{0}^{T-T_{0}}\langle\bar{h}(s, \cdot), \mathcal{L} \varphi\rangle d t \\
= & \left\langle\int_{0}^{T_{0}} w(T-s) h(t, \cdot) d t, \varphi\right\rangle+\left\langle\int_{T_{0}}^{T} w(T-s) \bar{h}\left(t-T_{0}, \cdot\right) d t, \varphi\right\rangle \\
= & \left\langle\int_{0}^{T} w(T-s) h(t, \cdot) d t, \varphi\right\rangle .
\end{aligned}
$$

Hence, for every $t>0$ and $\varphi \in \mathcal{D}(\mathcal{L})$,

$$
\int_{E} \varphi(x)\left(\int_{0}^{t} w(t-r) h(r, x) d r\right) \mu(d x)=\int_{E} h(t, x) \mathcal{L} \varphi(x) \mu(d x) .
$$

We now follow the uniqueness proof in [8, Theorem 2.3] (see also [13, Theorem 2.4]) to show that $h(t, x)=0$ in $L^{2}(E ; \mu)$ for every $t>0$. Let $H(\lambda, x):=\int_{0}^{\infty} e^{-\lambda t} h(t, x) d t, \lambda>0$, be the Laplace transform of $t \mapsto h(t, x)$. Note that, for every $\lambda>0, H(\lambda, \cdot) \in \mathbb{B}$ with $\|H(\lambda, \cdot)\|<\infty$; see (2.22). Taking the Laplace transform in $t$ on both sides of (2.35) yields that for every $\lambda>0$,

$$
\begin{aligned}
\frac{\phi(\lambda)}{\lambda} \int_{E} \varphi(x) H(\lambda, x) \mu(d x) & =\int_{E} \varphi(x) H(\lambda, x)\left(\int_{0}^{\infty} e^{-\lambda s} w(s) d s\right) \mu(d x) \\
& =\frac{1}{\lambda} \int_{E} H(\lambda, x) \mathcal{L} \varphi(x) \mu(d x) .
\end{aligned}
$$

That is, for every $\lambda>0$,

$$
\int_{E} H(\lambda, x)(\phi(\lambda)-\mathcal{L}) \varphi(x) \mu(d x)=0
$$

Denote by $\left\{G_{\alpha}^{0}: \alpha>0\right\}$ be the resolvent of the regular Dirichlet for $(\mathcal{E}, \mathcal{F})$. For each fixed $\lambda>0$ and $\eta \in L^{2}(E ; \mu)$, take $\varphi:=G_{\phi(\lambda)}^{0} \eta$, which is in $\mathcal{D}(\mathcal{L})$. Since $(\phi(\lambda)-\mathcal{L}) \varphi=\eta$, we deduce that $\int_{E} H(\lambda, x) \eta(x) \mu(d x)=0$ for every $\eta \in L^{2}(E ; \mu)$. Therefore $H(\lambda, \cdot)=0 \mu$-a.e. on $E$ for every $\lambda>0$. By the uniqueness of the Laplace transform and the fact that $t \mapsto h(t, x)$ is continuous in $L^{2}(E ; \mu)$, it follows that $(u-v)(t, \cdot)=h(t, \cdot)=0$ in $L^{2}(E ; \mu)$ for every $t>0$. Therefore, we conclude that $u(t, x)$ is the unique weak solution to $\partial_{t}^{w} u=\mathcal{L} u+f(t, x)$ on $\left(0, T_{0}\right] \times E$ with $u(0, x)=g(x)$. 


\section{Fundamental solutions to time fractional Poisson equations}

In this section, we assume that the Banach space $(\mathbb{B},\|\cdot\|)$ is a function space over a locally compact separable metric space $E$, where either $\mathbb{B}=L^{p}(E ; \mu)$ for some $\sigma$-finite measure $\mu$ with full support on $E$ and $p \geq 1$, or $\mathbb{B}=C_{\infty}(E)$, the space of continuous functions on $E$ that vanish at infinity. Let $\left\{P_{t}^{0} ; t \geq 0\right\}$ be a uniformly bounded and strongly continuous semigroup in $(\mathbb{B},\|\cdot\|)$. Recall that $S=\left\{S_{t}, \mathbb{P} ; t \geq 0\right\}$ is a driftless subordinator with infinite Lévy measure $\nu$ such that the subordinator $S_{t}$ has a bounded density function $\bar{p}(t, \cdot)$ for each $t>0$. As a particular case of Theorems 1.1 and 1.3. we know that

$$
u(t, x):=\int_{s=0}^{t} \int_{r=0}^{\infty} P_{r}^{0} f(s, \cdot)(x) \bar{p}(r, t-s) d r d s
$$

is the unique solution to the general time fractional Poisson equation (1.2) with $u(0, x)=0$ under suitable conditions on $f(t, x)$. We further assume the following assumption throughout this section.

Assumption 3.1 The uniformly bounded and strongly continuous semigroup $\left\{P_{t}^{0} ; t \geq 0\right\}$ in $(\mathbb{B}, \| \cdot$ $\|)$ has a density function $p^{0}(t, x, y)$ with respect to some $\sigma$-finite measure $\mu$ on $E$ with full support, such that

(i) $P_{t}^{0} f(x)=\int_{E} p^{0}(t, x, y) f(y) \mu(d y)$ for $f \in \mathbb{B}$;

(ii) for each $x, y \in E, t \mapsto p^{0}(t, x, y)$ is Borel measurable.

Under Assumption 3.1, $u(t, x)$ of (3.1) can be written as

$$
u(t, x)=\int_{0}^{t} \int_{E} q(t-s, x, y) f(s, y) \mu(d y) d s,
$$

where for $t>0$,

$$
q(t, x, y):=\int_{0}^{\infty} p^{0}(r, x, y) \bar{p}(r, t) d r .
$$

In other words, $q(t, x, y)$ is the fundamental solution for solving the time fractional Poisson equation (1.2) with zero initial value.

We know from [8, Theorem 2.3] (see also [13, Theorem 2.4]) that

$$
p(t, x, y):=\mathbb{E}\left[p^{0}\left(E_{t}, x, y\right)\right]
$$

is the fundamental solution of the homogenous time fractional equation (1.3).

Define $p(0, x, y)=0$ for $x \neq y$. This definition is reasonable in view of (3.3) as $E_{0}=0$ and $p^{0}(0, x, y) \mu(d y)=\delta_{\{x\}}(d y)$.

Recall that $G^{(S)}(t)$ is the potential density of the subordinator defined by (2.2).

Proposition 3.2 Suppose that $S_{r}$ has a bounded density $\bar{p}(r, \cdot)$ for each $r>0$ and that Assumption 3.1 holds. Then, for every $t>0$ and $x, y \in E$,

$$
\int_{0}^{t} q(s, x, y) d s=\int_{0}^{t} G^{(S)}(t-s) p(s, x, y) d s,
$$


Proof. First note that by Lemma 2.1, (3.2) and (3.3), there is a zero Lebesgue set $\mathcal{N}_{0} \subset(0, \infty)$ so that for every $t \in(0, \infty) \backslash \mathcal{N}_{0}$ and for every $x, y \in E$,

$$
p(t, x, y)=\int_{0}^{t} w(t-s) q(s, x, y) d s .
$$

Thus, by (3.5), the Fubini theorem and Lemma 2.2 , for every $t>0$,

$$
\begin{aligned}
\int_{0}^{t} G^{(S)}(t-s) p(s, x, y) d s & =\int_{s=0}^{t} G^{(S)}(t-s)\left(\int_{r=0}^{s} w(s-r) q(r, x, y) d r\right) d s \\
& =\int_{r=0}^{t} q(r, x, y)\left(\int_{s=r}^{t} w(s-r) G^{(S)}(t-s) d s\right) d r \\
& =\int_{r=0}^{t} q(r, x, y)\left(\int_{0}^{t-r} w(s) G^{(S)}(t-r-s) d s\right) d r \\
& =\int_{0}^{t} q(r, x, y) d r
\end{aligned}
$$

Recall that the subordinator $S$ is said to be special, if its Laplace exponent $\phi$ is a special Bernstein function; that is, $\lambda \mapsto \phi^{*}(\lambda):=\lambda / \phi(\lambda)$ is still a Bernstein function. In this case, let $S^{*}=\left\{S_{t}^{*} ; t \geq 0\right\}$ be the subordinator with the Laplace exponent $\phi^{*}(\lambda)$. We call $S^{*}$ the conjugate subordinator to $S$. Let $\nu^{*}$ be the Lévy measure of $S^{*}$ and $w^{*}(x):=\nu^{*}(x, \infty)$.

The aim of this section is to show that, when $S$ is a special subordinator and $S_{r}$ has a bounded density function $\bar{p}(r, \cdot)$ for each $r>0$, the fundamental solution $p(t, x, y)$ of the homogenous time fractional equation (1.3) has time-fractional derivative $\partial_{t}^{w^{*}} p(\cdot, x, y)(t)$, and

$$
q(t, x, y)=\partial_{t}^{w^{*}} p(\cdot, x, y)(t) \quad \text { for a.e. } t>0 \text { and } \mu \text {-a.e. } x \neq y \text { in } E \text {. }
$$

The following is a precise statement.

Theorem 3.3 Suppose that $S$ is a special subordinator, that $S_{r}$ has a bounded density $\bar{p}(r, \cdot)$ for each $r>0$ and that Assumption 3.1 holds. Denote by $\nu^{*}$ the Lévy measure of the conjugate subordinator $S^{*}$ to $S$ and set $w^{*}(x):=\nu^{*}(x, \infty)$. Let $q(t, x, y)$ and $p(t, x, y)$ be defined as in (3.2) and (3.3), respectively. Then

$$
\int_{0}^{t} q(s, x, y) d s=\int_{0}^{t} w^{*}(t-s) p(s, x, y) d s \quad \text { for } t>0
$$

which is finite for $\mu$-a.e. $x \in E$ and $\mu$-a.e. $y \in E \backslash\{x\}$. Hence for any such $x \neq y$ so that (3.6) holds,

$$
\partial_{t}^{w^{*}} p(\cdot, x, y)(t):=\frac{d}{d t} \int_{0}^{t} w^{*}(t-s) p(s, x, y) d s \quad \text { exists for a.e. } t>0
$$

and

$$
q(t, x, y)=\partial_{t}^{w^{*}} p(\cdot, x, y)(t) \quad \text { for a.e. } t>0 .
$$


Proof. Since $S$ is a special subordinator and $\nu$ is infinite, $w^{*}(t)=G^{(S)}(t)$ (see [36, Corollary 11.8]). Thus (3.6) follows immediately from this and Proposition 3.2.

We now show the finiteness of (3.6). Let $M=\sup _{t>0}\left\|P_{t}^{0}\right\|$. For every $t>0$ and $f \in \mathbb{B}$, by (3.1), (3.2) and Fubini's theorem,

$$
\begin{aligned}
\left\|\int_{E}\left(\int_{0}^{t} q(s, \cdot, y) d s\right) f(y) \mu(d y)\right\| & \leq \int_{0}^{t} \int_{0}^{\infty}\left\|P_{r}^{0} f\right\| \bar{p}(r, s) d r d s \\
& \leq M\|f\| \int_{0}^{t} \int_{0}^{\infty} \bar{p}(r, s) d r d s=M\|f\| G^{(S)} \mathbf{1}_{(0, t]}(0)<\infty .
\end{aligned}
$$

Hence by (3.6), for $\mu$-a.e. $x \in E$ and $\mu$-a.e. $y \in E \backslash\{x\}$,

$$
\int_{0}^{t} q(s, x, y) d s=\int_{0}^{t} w^{*}(t-s) p(s, x, y) d s<\infty \quad \text { for every } t>0 .
$$

This proves the theorem.

\section{Two-sided estimates}

\subsection{Density estimates for subordinators}

In this subsection, we assume that the driftless subordinator $S=\left\{S_{t}, \mathbb{P} ; t \geq 0\right\}$ satisfies the following assumptions.

Assumption 4.1 (i) The Laplace exponent $\phi$ of $S$ satisfies that

$$
c_{1} \kappa^{\beta_{1}} \leq \frac{\phi(\kappa \lambda)}{\phi(\lambda)} \leq c_{2} \kappa^{\beta_{2}} \quad \text { for all } \lambda>0 \text { and } \kappa \geq 1
$$

where $0<\beta_{1}<\beta_{2}<1$. Without loss of generality, we assume $\phi(1)=1$.

(ii) The Lévy measure $\nu(d z)$ of $S$ has a density function $\nu(z)$ with respect to the Lebesgue measure such that

$$
t \mapsto t \nu(t) \text { is non-increasing on }(0, \infty) \text {. }
$$

We make some remarks on the assumption above.

Remark 4.2 (i) Under (4.1), the Lévy measure $\nu$ of $S$ is infinite as $\nu(0, \infty)=\lim _{\lambda \rightarrow \infty} \phi(\lambda)=\infty$, excluding compound Poisson processes.

(ii) Condition (4.2) is equivalent to saying that $\mathbb{P}\left(S_{1} \in d t\right)$ is self-decomposable (see [36, Definition 5.14 and Proposition 5.17]).

(iii) Under (4.1) and (4.2), it follows from the proof of [26, Proposition 2.5] that $\nu(t) \simeq t^{-1} \phi\left(t^{-1}\right)$ for all $t>0$.

(iv) According to [35, Theorems 27.13 and 28.4(ii)], (4.1) and (4.2) together imply that $S_{r}$ has a bounded density $\bar{p}(r, t)$ so that $\mathbb{P}\left(S_{r} \in d t\right)=\bar{p}(r, t) d t$; moreover, $t \mapsto \bar{p}(r, t)$ is smooth for any $r>0$. 
We now mention two large classes of subordinators that satisfy both (4.1) and (4.2).

Example 4.3 (1) Let $0<\beta_{1}<\beta_{2}<1$. Suppose a positive function $\kappa(\beta, t)$ defined on $\left[\beta_{1}, \beta_{2}\right] \times$ $(0, \infty)$ satisfies that $c_{1}^{-1} \leq \kappa(\beta, t) \leq c_{1}$ for all $(\beta, t) \in\left[\beta_{1}, \beta_{2}\right] \times(0, \infty)$ and some constant $c_{1} \geq 1$, and that $t \mapsto \kappa(\beta, t)$ is non-increasing on $(0, \infty)$ for any fixed $\beta \in\left[\beta_{1}, \beta_{2}\right]$. Define

$$
\phi(\lambda):=\int_{0}^{\infty}\left(1-e^{-\lambda t}\right) \nu(t) d t
$$

where

$$
\nu(t):=t^{-1} \int_{\beta_{1}}^{\beta_{2}} \kappa(\beta, t) t^{-\beta} \mu_{I}(d \beta)
$$

and $\mu_{I}$ is a finite measure on $I:=\left[\beta_{1}, \beta_{2}\right]$. Then clearly (4.2) holds. Furthermore, since

$$
\phi(\lambda) \simeq \int_{\beta_{1}}^{\beta_{2}} \int_{0}^{\infty}\left(1-e^{-\lambda t}\right) t^{-1-\beta} d t \mu_{I}(d \beta)=\int_{\beta_{1}}^{\beta_{2}}\left(\int_{0}^{\infty}\left(1-e^{-s}\right) s^{-1-\beta} d s\right) \lambda^{\beta} \mu_{I}(d \beta),
$$

it is easy to see that (4.1) holds too. In particular, stable subordinators, which correspond to $\mu_{I}$ being a Dirac measure $\delta_{\{\beta\}}$ for some $\beta \in(0,1)$ and $\kappa(\beta, t) \equiv c_{0}$ for all $t>0$ for some positive constant $c_{0}$, and a larger class of mixtures of stable subordinators satisfy both (4.1) and (4.2). For more related examples, see [35, Examples 15.13-15.15] and the references therein.

(2) A function $f:(0, \infty) \rightarrow[0, \infty)$ is said to be completely monotone, if $f$ is of class $C^{\infty}$ and $(-1)^{n} f^{(n)} \geq 0$ on $(0, \infty)$ for every integer $n \geq 0$. A Bernstein function is said to be a complete Bernstein function, if its Lévy measure has a completely monotone density with respect to the Lebesgue measure. A sufficient condition on $\phi$ which implies (4.2) is that $\phi$ is a Thorin-Bernstein function; that is, both $\phi(\lambda)$ and $\lambda \phi^{\prime}(\lambda)$ are complete Bernstein functions. In this case, both $t \mapsto \nu(t)$ and $t \mapsto t \nu(t)$ are completely monotone. (See [36, Definition 8.1, Theorem 8.2(iv) and Theorem $6.2]$.

The main result of this subsection is as follows.

Theorem 4.4 Let $S$ be a subordinator such that (4.1) and (4.2) are satisfied. Denote by $\bar{p}(r, t)$ the associated transition density. Then, for each $L>0$, there exist constants $c_{i}:=c_{i, L} \geq 1(i=1,2,3)$ such that

$$
\frac{1}{c_{1} t} r \phi\left(t^{-1}\right) \leq \bar{p}(r, t) \leq \frac{c_{1}}{t} r \phi\left(t^{-1}\right) \quad \text { for every positive } r, t \text { with } r \phi\left(t^{-1}\right) \leq L
$$

and

$$
\frac{1}{c_{2} t} e^{-c_{3} t\left(\phi^{\prime}\right)^{-1}(t / r)} \leq \bar{p}(r, t) \leq \frac{c_{2}}{t} e^{-c_{3}^{-1} t\left(\phi^{\prime}\right)^{-1}(t / r)} \quad \text { for every positive } r, t \text { with } r \phi\left(t^{-1}\right) \geq L
$$

where $\left(\phi^{\prime}\right)^{-1}(\lambda):=\inf \left\{s>0: \phi^{\prime}(s) \leq \lambda\right\}$ for all $\lambda \geq 0$.

To prove Theorem 4.4, we will make use of the following scaled processes. For $r>0$, define

$$
\phi^{r}(\lambda):=\frac{\phi\left(\lambda r^{-1}\right)}{\phi\left(r^{-1}\right)}, \quad \lambda>0 .
$$


Note that $\phi^{r}(1)=1$ for all $r>0$. Since

$$
\phi^{r}(\lambda)=\frac{\phi\left(\lambda r^{-1}\right)}{\phi\left(r^{-1}\right)}=\int_{0}^{\infty}\left(1-e^{-\lambda t / r}\right) \phi\left(r^{-1}\right)^{-1} \nu(t) d t=\int_{0}^{\infty}\left(1-e^{-\lambda s}\right) \phi\left(r^{-1}\right)^{-1} r \nu(r s) d s,
$$

$\phi^{r}$ is a driftless Bernstein function with Lévy density $\nu^{r}$ given by $\nu^{r}(t)=r \phi\left(r^{-1}\right)^{-1} \nu(r t)$. It is obvious that (4.2) is satisfied, and (4.1) holds for all $\phi^{r}$ with the same constants, i.e.,

$$
c_{1} \kappa^{\beta_{1}} \leq \frac{\phi^{r}(\kappa \lambda)}{\phi^{r}(\lambda)} \leq c_{2} \kappa^{\beta_{2}} \quad \text { for any } \lambda, r>0 \text { and } \kappa \geq 1 .
$$

In particular, according to [26, Proposition 2.5] (and its proof) again, (4.5) implies that

$$
\nu^{r}(t) \simeq \phi^{r}\left(t^{-1}\right) t^{-1}, \quad t, r>0 .
$$

Let $S^{r}=\left\{S_{t}^{r}, \mathbb{P} ; t \geq 0\right\}$ be a subordinator with the Laplace exponent $\phi^{r}$. Since

$$
\mathbb{E}\left[e^{-\lambda S_{t}^{r}}\right]=e^{-\frac{t}{\phi\left(r^{-1}\right)} \phi\left(\lambda r^{-1}\right)}=\mathbb{E}\left[e^{-\lambda r^{-1} S_{t / \phi\left(r^{-1}\right)}}\right],
$$

$S^{r}$ is identical in law to the process $\left\{r^{-1} S_{t / \phi\left(r^{-1}\right)}, \mathbb{P} ; t \geq 0\right\}$. In particular, $\frac{1}{\phi^{-1}(1 / t)} S_{1}^{1 / \phi^{-1}(1 / t)}$ enjoys the same law as that of $S_{t}$, so the density $q^{r}$ of $S_{1}^{r}$ has the identity

$$
\phi^{-1}(1 / t) q^{1 / \phi^{-1}(1 / t)}\left(u \phi^{-1}(1 / t)\right)=\bar{p}(t, u), \quad t, r>0 .
$$

We have the following strictly unimodal property and an upper bound for $\bar{p}(r, t)$.

Lemma 4.5 Under (4.1), and (4.2), the following hold.

(i) There exists a constant $a_{r}>0$ such that for all $r>0, t \mapsto \bar{p}(r, t)$ is strictly increasing on $\left[0, a_{r}\right)$ and $t \mapsto \bar{p}(r, t)$ is strictly decreasing on $\left(a_{r}, \infty\right)$. Moreover, there exists a constant $c_{1} \geq 1$ such that $c_{1}^{-1} / \phi^{-1}(1 / r) \leq a_{r} \leq c_{1} / \phi^{-1}(1 / r)$ for all $r>0$.

(ii) There exists a constant $c_{2}>0$ such that for all $r, t>0$,

$$
\bar{p}(r, t) \leq c_{2} \phi^{-1}(1 / r) .
$$

Proof. (i) We first note that, since $\lim _{t \downarrow 0} t \nu(t)=\infty$ and $\int_{0}^{1} t \nu(t) d t<\infty$ by (4.1), (4.2) and Remark 4.2(iii), $S_{r}$ is of type $I_{6}$ in [34] for each $r>0$ (see [34, p. 275]). Thus, according to 34, Theorem 1.4] and [40, Corollary 1], $\mathbb{P}\left(S_{r} \in d t\right)$ is strictly unimodal with mode $a_{r} \geq 0$ on $[0, \infty)$; that is, $\mathbb{P}\left(S_{r} \in d t\right)=c_{r} \delta_{a_{r}}(d t)+\widehat{p}(r, t) d t$, where $c_{r} \geq 0, t \mapsto \widehat{p}(r, t)$ is strictly increasing on $\left[0, a_{r}\right]$ and $t \mapsto \widehat{p}(r, t)$ is strictly decreasing on $\left[a_{r}, \infty\right)$. Since $\mathbb{P}\left(S_{r} \in d t\right)=\bar{p}(r, t) d t$, we have $c_{r}=0$ and so $\widehat{p}(r, t)=\bar{p}(r, t)$.

We next show that $a_{r} \asymp 1 / \phi^{-1}(1 / r)$ on $(0, \infty)$ using the above scaled processes $S^{r}$. We first claim that the infinitely divisible random variables $S_{1}^{r}$ 's have their modes $b_{r}$ 's such that $b_{r} \simeq 1$ for all $r>0$. First of all, since $t \mapsto t \nu^{r}(t)=r \phi\left(r^{-1}\right)^{-1} t \nu(r t)$ is non-increasing on $(0, \infty), S_{1}^{r}$ 's are self-decomposable by Remark 4.2(ii); moreover, $S_{1}^{r}$ 's are of type $I_{6}$ in 34 because of (4.5), (4.6) and Remark4.2(iii). Thus, according to [34, Theorem 6.1 (ii) and (vi)], we have that $b_{r}^{-1} \int_{0}^{b_{r}} \nu^{r}(t) t d t>1$ and $b_{r}>\sup \left\{t: \nu^{r}(t) t \geq 1\right\}$. Using $\phi^{r}(1)=1$, (4.5) and (4.6) to these two inequalities, we get

$$
b_{r}>\sup \left\{u: \nu^{r}(u) u \geq 1\right\} \geq c_{1} \sup \left\{u: \phi^{r}\left(u^{-1}\right) \geq 1\right\} \geq c_{1}
$$


and

$$
\begin{aligned}
1 & <b_{r}^{-1} \int_{0}^{b_{r}} \nu^{r}(t) t d t \leq c_{2} b_{r}^{-1} \int_{0}^{b_{r}} \phi^{r}\left(t^{-1}\right) d t=c_{2} \phi^{r}\left(b_{r}^{-1}\right) b_{r}^{-1} \int_{0}^{b_{r}} \frac{\phi^{r}\left(t^{-1}\right)}{\phi^{r}\left(b_{r}^{-1}\right)} d t \\
& \leq c_{3} \phi^{r}\left(b_{r}^{-1}\right) b_{r}^{-1} \int_{0}^{b_{r}}\left(\frac{b_{r}}{t}\right)^{\beta_{2}} d t=c_{4} \phi^{r}\left(b_{r}^{-1}\right) .
\end{aligned}
$$

Therefore, by the inequalities above and (4.5),

$$
c_{1} \leq b_{r} \leq \frac{1}{\phi_{r}^{-1}\left(1 / c_{4}\right)}=\frac{\phi_{r}^{-1}(1)}{\phi_{r}^{-1}\left(1 / c_{4}\right)} \leq c_{5} .
$$

We have proved the claim. Now, using (4.7), we conclude that $a_{r} \simeq 1 / \phi^{-1}(1 / r)$ on $(0, \infty)$.

(ii) Recall that $q^{r}$ is the density of $S_{1}^{r}$. Let

$$
x^{r}(\lambda):=\int_{0}^{\infty}(1-\cos (\lambda t)) \nu^{r}(t) d t
$$

and

$$
y^{r}(\lambda):=\int_{0}^{\infty} \sin (\lambda t) \nu^{r}(t) d t
$$

which are finite by (4.5) and (4.6). (4.5) and (4.6) also imply that the characteristic exponent of $S_{t}^{r}$ is

$$
\psi^{r}(\lambda):=x^{r}(\lambda)-i y^{r}(\lambda)=\int_{0}^{\infty}\left(1-e^{i \lambda t}\right) \nu^{r}(t) d t, \quad \lambda>0 .
$$

Using the inequality $(1-\cos s) \geq(1-\cos 1) s^{2}$ for any $s \in(0,1)$, (4.5) and (4.6), we have

$$
\begin{aligned}
x^{r}(\lambda) & \geq(1-\cos 1) \int_{0}^{1 / \lambda}(\lambda t)^{2} \nu^{r}(t) d t \geq c_{1} \lambda^{2} \phi^{r}(\lambda) \int_{0}^{1 / \lambda} t \frac{\phi^{r}(1 / t)}{\phi^{r}(\lambda)} d t \\
& \geq c_{2} \lambda^{2} \phi^{r}(\lambda) \int_{0}^{1 / \lambda} t(t \lambda)^{-\beta_{1}} d t \geq c_{3} \phi^{r}(\lambda) .
\end{aligned}
$$

In particular, $e^{-\psi^{r}} \in L^{1}(\mathbb{R} ; d x)$, so by applying the Fourier inversion formula and then using (4.5) we can bound $q^{r}$ as

$$
\begin{aligned}
q^{r}(z) & =\frac{1}{2 \pi} \int_{\mathbb{R}} e^{-\lambda z-\psi^{r}(z)} d z=\frac{1}{2 \pi} \int_{\mathbb{R}} e^{i\left(y^{r}(\lambda)-\lambda z\right)-x^{r}(\lambda)} d \lambda \\
& \leq \frac{1}{\pi} \int_{0}^{\infty} e^{-x^{r}(\lambda)} d \lambda \leq \frac{1}{2 \pi}\left(1+\int_{1}^{\infty} e^{-c_{4} \lambda^{\beta_{1}}} d \lambda\right) .
\end{aligned}
$$

Thus, $q^{r}$ 's are uniformly bounded. Now, the second assertion follows from this and (4.7).

To establish two-sided estimates of $\bar{p}(r, t)$, we further need the following two statements from [13. Lemma 3.1 and Proposition 3.3], respectively.

Lemma 4.6 ([13, Lemma 3.1]) Let $\phi$ be a Bernstein function such that (4.1) is satisfied. Then there exists a constant $C_{*} \geq 1$ such that the following holds

$$
\lambda \phi^{\prime}(\lambda) \leq \phi(\lambda) \leq C_{*} \lambda \phi^{\prime}(\lambda), \quad \lambda>0 .
$$


In particular, there exist constants $c_{i}>0(i=3,4,5,6)$ such that

$$
c_{3} \kappa^{1-\beta_{2}} \leq \frac{\phi^{\prime}(\lambda)}{\phi^{\prime}(\kappa \lambda)} \leq c_{4} \kappa^{1-\beta_{1}}, \quad \lambda>0, \kappa \geq 1
$$

and

$$
c_{5} \kappa^{1 /\left(1-\beta_{1}\right)} \leq \frac{\left(\phi^{\prime}\right)^{-1}(\lambda)}{\left(\phi^{\prime}\right)^{-1}(\kappa \lambda)} \leq c_{6} \kappa^{1 /\left(1-\beta_{2}\right)}, \quad \lambda>0, \kappa \geq 1
$$

Proposition 4.7 ([13, Proposition 3.3]) Let $S=\left\{S_{t}, \mathbb{P} ; t \geq 0\right\}$ be a subordinator whose Laplace exponent $\phi$ satisfies assumption (4.1). Then, we have

(i) There are constants $c_{1}, c_{2}>0$ such that for all $r, t \geq 0$,

$$
\mathbb{P}\left(S_{r} \geq t\left(1+\operatorname{er} \phi\left(t^{-1}\right)\right) \leq c_{1} r \phi\left(t^{-1}\right)\right.
$$

and

$$
\mathbb{P}\left(S_{r} \geq t\right) \geq 1-e^{-c_{2} r \phi\left(t^{-1}\right)} .
$$

In particular, for each $L>0$, there exist constants $c_{1, L}, c_{2, L}>0$ such that for all $r \phi\left(t^{-1}\right) \leq L$,

$$
c_{1, L} r \phi\left(t^{-1}\right) \leq \mathbb{P}\left(S_{r} \geq t\right) \leq c_{2, L} r \phi\left(t^{-1}\right) .
$$

(ii) There is a constant $c_{1}>0$ such that for all $r, t>0$,

$$
\mathbb{P}\left(S_{r} \leq t\right) \leq \exp \left(-c_{1} r \phi \circ\left[\left(\phi^{\prime}\right)^{-1}\right](t / r)\right) \leq \exp \left(-c_{1} t\left(\phi^{\prime}\right)^{-1}(t / r)\right) .
$$

Moreover, there is a constant $c_{0}>0$ such that for each $L>0$, there exists a constant $c_{c_{0}, L}>0$ so that for $r \phi\left(t^{-1}\right)>L$

$$
\mathbb{P}\left(S_{r} \leq t\right) \geq c_{c_{0}, L} \exp \left(-c_{0} r \phi \circ\left[\left(\phi^{\prime}\right)^{-1}\right](t / r)\right) \geq c_{c_{0}, L} \exp \left(-c_{0} C_{*} t\left(\phi^{\prime}\right)^{-1}(t / r)\right),
$$

where $C_{*}>0$ is an absolute constant only depending on $\phi$.

Now, we are in a position to present the

Proof of Theorem 4.4. (1) By Lemma 4.5(i), there exists a constant $L \geq 1$ such that for every fixed $r>0, t \mapsto \bar{p}(r, t)$ is strictly decreasing for $t \geq 1 / \phi^{-1}(1 /(L r))$ and $t \mapsto \bar{p}(r, t)$ is strictly increasing for $t \leq 1 / \phi^{-1}(L / r)$.

By Proposition 4.7(i), for any $r, t>0$ with $r \phi\left(t^{-1}\right) \leq 1 / L$ and for any $c>1$,

$$
\begin{aligned}
\bar{p}(r, t) & \geq \frac{1}{(c-1) t} \int_{t}^{c t} \bar{p}(r, s) d s=\frac{1}{(c-1) t}\left(\mathbb{P}\left(S_{r}>t\right)-\mathbb{P}\left(S_{r}>c t\right)\right) \\
& \geq \frac{r}{(c-1) t}\left(c_{1, L} \phi\left(t^{-1}\right)-c_{2, L} \phi\left((c t)^{-1}\right)\right) .
\end{aligned}
$$

In the first inequality above we have used the fact that $s \mapsto \bar{p}(r, s)$ is decreasing on $(t, c t)$. Thus, by (4.1), taking $c>0$ large enough, we arrive at

$$
\bar{p}(r, t) \geq \frac{c_{1, L}}{2(c-1) t} r \phi\left(t^{-1}\right) \quad \text { for all } r \phi\left(t^{-1}\right) \leq 1 / L .
$$


Similarly, since $s \mapsto \bar{p}(r, s)$ is decreasing on $(t / 2, \infty)$ if $r \phi\left(2 t^{-1}\right) \leq 1 / L$, we have that for $r \phi\left(2 t^{-1}\right) \leq$ $1 / L$

$$
\bar{p}(r, t) \leq \frac{2}{t} \int_{t / 2}^{t} \bar{p}(r, s) d s=\frac{2}{t}\left(\mathbb{P}\left(S_{r}>t\right)-\mathbb{P}\left(S_{r}>t / 2\right)\right) \leq \frac{2}{t} c_{2, L} r \phi\left(t^{-1}\right) .
$$

On the other hand, according to Proposition 4.7(ii), for any $r, t>0$ with $r \phi\left(t^{-1}\right)>L$

$$
\bar{p}(r, t) \geq \frac{1}{t} \int_{0}^{t} \bar{p}(r, s) d s=\frac{1}{t} \mathbb{P}\left(S_{r} \leq t\right) \geq \frac{1}{t} c_{c_{3}, L} \exp \left(-c_{4} t\left(\phi^{\prime}\right)^{-1}(t / r)\right) .
$$

In the first inequality above we have used the fact that $s \mapsto \bar{p}(r, s)$ is increasing on $(0, t)$. Similarly, since $s \mapsto \bar{p}(r, s)$ is increasing on $(0,2 t)$ if $r \phi\left((2 t)^{-1}\right)>L$, we have that for any $r, t>0$ with $r \phi\left((2 t)^{-1}\right)>L$,

$$
\bar{p}(r, t) \leq \frac{1}{t} \int_{t}^{2 t} \bar{p}(r, s) d s=\frac{1}{t}\left(\mathbb{P}\left(S_{r} \leq 2 t\right)-\mathbb{P}\left(S_{r} \leq t\right)\right) \leq \frac{1}{t} \exp \left(-c_{5} t\left(\phi^{\prime}\right)^{-1}(t / r)\right) .
$$

Hence, combining above estimates and using (4.1), we have that there exists a constant $C>1$ such that

$$
\bar{p}(r, t) \simeq \frac{1}{t} r \phi\left(t^{-1}\right) \quad \text { for every positive } r, t \text { with } r \phi\left(t^{-1}\right) \leq C^{-1}
$$

and

$$
\frac{c_{6, C}}{t} e^{-c_{7} t\left(\phi^{\prime}\right)^{-1}(t / r)} \leq \bar{p}(r, t) \leq \frac{1}{t} e^{-c_{8} t\left(\phi^{\prime}\right)^{-1}(t / r)} \quad \text { for every positive } r, t \text { with } r \phi\left(t^{-1}\right) \geq C .
$$

(2) Next, we consider the case that $t, r>0$ satisfying $C^{-1} \leq r \phi\left(t^{-1}\right) \leq C$. Since

$$
r \cdot \phi^{\prime} \circ\left[\left(\phi^{\prime}\right)^{-1}\right](t / r) \cdot\left(\phi^{\prime}\right)^{-1}(t / r)=t \cdot\left(\phi^{\prime}\right)^{-1}(t / r),
$$

by (4.8) we have

$$
t \cdot\left(\phi^{\prime}\right)^{-1}(t / r) \simeq \frac{\phi \circ\left[\left(\phi^{\prime}\right)^{-1}\right]\left(t \phi\left(t^{-1}\right)\right)}{\phi\left(t^{-1}\right)}=\frac{\phi \circ\left[\left(\phi^{\prime}\right)^{-1}\right]\left(\frac{\phi\left(t^{-1}\right)}{t^{-1}}\right)}{\phi \circ\left[\left(\phi^{\prime}\right)^{-1}\right]\left(\phi^{\prime}\left(t^{-1}\right)\right)} \simeq 1 .
$$

Using Proposition 4.5 and (4.13), we get

$$
\bar{p}(r, t) \leq c_{1} \phi^{-1}(1 / r) \leq c_{1} \phi^{-1}(C \phi(1 / t)) \leq c_{2} t^{-1} \leq c_{3} t^{-1}\left[C r \phi\left(t^{-1}\right) \wedge e^{-c_{4} t\left(\phi^{\prime}\right)^{-1}(t / r)}\right] .
$$

For lower bound of $\bar{p}(r, t)$ on $C^{-1} \leq r \phi\left(t^{-1}\right) \leq C$, we use the unimodality of $\bar{p}(r, t)$ and (4.11)(4.13), and get

$$
\bar{p}(r, t) \geq \bar{p}\left(r, 1 / \phi^{-1}(C / r)\right) \wedge \bar{p}\left(r, 1 / \phi^{-1}(r / C)\right) \geq c_{5} t^{-1} \geq \frac{c_{5}}{2 t}\left(C^{-1} r \phi\left(t^{-1}\right)+e^{-t\left(\phi^{\prime}\right)^{-1}(t / r)}\right) .
$$

Therefore, combining (4.11), (4.12), (4.14) and (4.15) together, we have proved the theorem. 


\subsection{Two-sided estimates of the fundamental solution}

Suppose that $0<\alpha_{1} \leq \alpha_{2}<\infty$. We say that a non-decreasing function $\Psi:(0, \infty) \rightarrow(0, \infty)$ satisfies the weak scaling property with $\left(\alpha_{1}, \alpha_{2}\right)$ if there exist constants $c_{1}$ and $c_{2}>0$ such that

$$
c_{1}(R / r)^{\alpha_{1}} \leq \Psi(R) / \Psi(r) \leq c_{2}(R / r)^{\alpha_{2}} \text { for all } 0<r \leq R<\infty .
$$

We say that a family of non-decreasing functions $\left\{\Psi_{x}\right\}_{x \in \Lambda}$ satisfies the weak scaling property uniformly with $\left(\alpha_{1}, \alpha_{2}\right)$ if each $\Psi_{x}$ satisfies the weak scaling property with constants $c_{1}, c_{2}>0$ and $0<\alpha_{1} \leq \alpha_{2}<\infty$ independent of the choice of $x \in \Lambda$.

In this subsection, let $(E, d, \mu)$ be a locally compact separable metric measure space such that $\mu$ is a Radon measure on $(E, d)$ with full support. For $x \in E$ and $r \geq 0$, define

$$
V(x, r)=\mu(B(x, r)) .
$$

We further assume that for each $x \in E, V(x, \cdot)$ satisfies the weak scaling property uniformly with $\left(d_{1}, d_{2}\right)$ for some $d_{2} \geq d_{1}>0$; that is, for any $0<r \leq R$ and $x \in E$,

$$
c_{1}\left(\frac{R}{r}\right)^{d_{1}} \leq \frac{V(x, R)}{V(x, r)} \leq c_{2}\left(\frac{R}{r}\right)^{d_{2}}
$$

Note that (4.17) is equivalent to the so-called volume doubling and reverse volume doubling conditions.

Let $\left\{P_{t}^{0} ; t \geq 0\right\}$ be a uniformly bounded and strongly continuous semigroup on $L^{p}(E ; \mu)$ with $p \geq 1$ or on $C_{\infty}(E)$ having a density $p^{0}(t, x, y)$ with respect to $\mu$. Let $\left\{S_{t}, \mathbb{P} ; t \geq 0\right\}$ be the driftless subordinator satisfying (4.1) and (4.2). In particular, $S_{r}$ has a density $\bar{p}(r, t)$ so that $\bar{p}(r, t)$ obeys two-sided estimates as these in Theorem 4.4.

The purpose of this part is to establish two-sided estimates for the fundamental solution $q(t, x, y)$ for the time fractional Poisson equation given by (3.2), i.e.,

$$
q(t, x, y)=\int_{0}^{\infty} p^{0}(r, x, y) \bar{p}(r, t) d r .
$$

We adopt the setting of [13], where two-sided estimates on the fundamental solution $p(r, x, y)$ of the homogenous time fractional equation were derived. We will consider estimates for $q(t, x, y)$ when $\left\{P_{t}^{0} ; t \geq 0\right\}$ is associated with pure jump processes and diffusion processes, respectively. Since the arguments are partly similar to these in [13, Sections 4 and 5], we just present main results on two-sided estimates for the fundamental solution for the time fractional Poisson equation, and then give some intuitive explanations on these estimates and present the proof of Theorem 1.4. Full proofs of theorems are postponed to the appendix of this paper.

\subsubsection{Pure jump case}

We first consider the case that $\left\{P_{t}^{0} ; t \geq 0\right\}$ is corresponding to a strong Markov processes with pure jumps, where the associated transition density $p^{0}(t, x, y)$ enjoys the following two-sided estimates:

$$
p^{0}(t, x, y) \simeq \frac{1}{V\left(x, \Phi^{-1}(t)\right)} \wedge \frac{t}{V(x, d(x, y)) \Phi(d(x, y))}=: \bar{p}^{0}(t, x, d(x, y)), \quad t>0, x, y \in E
$$


Here $\Phi:[0,+\infty) \rightarrow[0,+\infty)$ is a strictly increasing function with $\Phi(0)=0$ that satisfies the weak scaling property with $\left(\alpha_{1}, \alpha_{2}\right)$, i.e., (4.16) is satisfied. The most typical examples are the heat kernels for $\alpha$-stable-like processes on $d$-sets, in which $\Phi(r)=r^{\alpha}$ and $V(x, r)=r^{d}$ for all $x \in E$ and $r>0$. Further examples of pure jump processes with heat kernel estimates can be referred to [10, 11] and the references therein.

Theorem 4.8 We assume that Assumption 4.1 holds. Let $q(t, x, y)$ be given by (3.2). Suppose that $p^{0}(t, x, y)$ enjoys two-sided estimates (4.18). Then we have the following two statements:

(i) If $\Phi(d(x, y)) \phi\left(t^{-1}\right) \leq 1$, then

$$
q(t, x, y) \simeq \frac{\phi\left(t^{-1}\right)}{t} \int_{\Phi(d(x, y))}^{2 / \phi\left(t^{-1}\right)} \frac{r}{V\left(x, \Phi^{-1}(r)\right)} d r .
$$

(ii) If $\Phi(d(x, y)) \phi\left(t^{-1}\right) \geq 1$, then

$$
q(t, x, y) \simeq \frac{1}{t \phi\left(t^{-1}\right)^{2} V(x, d(x, y)) \Phi(d(x, y))} .
$$

Proof of Theorem 1.4(i). By [20, Theorem 3.2], our assumption on the estimates (1.10) of Markovian transition kernel $p^{0}(t, x, y)$ implies that $V(x, r) \simeq r^{d}$. Thus, $\Phi(r)=r^{\alpha}, V(x, r) \simeq r^{d}$ and $\phi(r)=r^{\beta}$ in this case, Therefore, the condition $\Phi(d(x, y)) \phi\left(t^{-1}\right) \leq 1$ is equivalent to $d(x, y) \leq t^{\beta / \alpha}$. The conclusion of Theorem 1.4(i) is then an easy consequence of Theorem 4.8 .

\subsubsection{Diffusion case}

We next consider the case that the strong Markov process $X$ corresponding to $\left\{P_{t}^{0} ; t \geq 0\right\}$ is a conservative diffusion; that is, it has continuous sample paths and infinite lifetime. We assume that the heat kernel $p^{0}(t, x, y)$ of the diffusion $X$ with respect to $\mu$ exists and enjoys the following two-sided estimates

$$
p^{0}(t, x, y) \asymp \frac{1}{V\left(x, \Phi^{-1}(t)\right)} \exp (-m(t, d(x, y)))=: \bar{p}^{0}(t, x, d(x, y)), \quad t>0, x, y \in E .
$$

Here, $\Phi:[0,+\infty) \rightarrow[0,+\infty)$ is a strictly increasing function with $\Phi(0)=0$, and satisfies the weak scaling property with $\left(\alpha_{1}, \alpha_{2}\right)$ such that $\alpha_{2} \geq \alpha_{1}>1$ in (4.16); the function $m(t, r)$ is strictly positive for all $t, r>0$, non-increasing on $t \in(0, \infty)$ for fixed $r>0$, and determined by

$$
\frac{t}{m(t, r)} \simeq \Phi\left(\frac{r}{m(t, r)}\right), \quad t, r>0
$$

In all the literature we know, for example [19, Section 7], [21, Page 1217-1218] and [22]), estimate (4.20) was established under assumptions that include $(E, d)$ being connected and satisfying the chain condition (that is, there exists a constant $C>0$ such that, for any $x, y \in M$ and for any $n \in \mathbb{N}$, there exists a sequence $\left\{x_{i}\right\}_{i=0}^{n} \subset E$ such that $x_{0}=x, x_{n}=y$ and $d\left(x_{i}, x_{i+1}\right) \leq C d(x, y) / n$ for all $i=0,1, \cdots, n-1)$. However, given the estimate (4.20) on $p_{0}(t, x, y)$, we do not need to assume the connectedness nor the chain condition on $(E, d)$ in arguments below for the estimates 
of $q(t, x, y)$. Since (4.16) holds with $\alpha_{1}>1$ and (4.21) is satisfied, there are constants $c_{1}, c_{2}>0$ such that for all $r>0$,

$$
c_{1}\left(\frac{T}{t}\right)^{-1 /\left(\alpha_{1}-1\right)} \leq \frac{m(T, r)}{m(t, r)} \leq c_{2}\left(\frac{T}{t}\right)^{-1 /\left(\alpha_{2}-1\right)}, \quad 0<t \leq T .
$$

On the other hand, due to (4.21),

$$
m(\Phi(r), r) \simeq 1, \quad r>0 .
$$

Using this and the fact that $m(\cdot, r)$ is non-increasing, we have that for every $c_{3}>0$ there exists $c_{4} \geq 1$ such that

$$
\frac{c_{4}^{-1}}{V\left(x, \Phi^{-1}(t)\right)} \leq p^{0}(t, x, y) \leq \frac{c_{4}}{V\left(x, \Phi^{-1}(t)\right)} \quad \text { when } \Phi(d(x, y)) \leq c_{3} t
$$

This means that both (4.18) and (4.20) enjoy the same form for near diagonal heat kernel estimates. Different from (4.18) where off-diagonal heat kernel estimates are due to jump systems of the corresponding jump processes, in (4.20) off-diagonal heat kernel estimates are characterized by diffusion property and the so-called chain arguments. The most typical examples are the subGaussian heat kernels for Brownian motion on some fractals, in which $\Phi(r)=r^{d_{w}}, V(x, r)=r^{d}$ and $m(t, r)=\left(r^{d_{w}} / t\right)^{1 /\left(d_{w}-1\right)}$ for all $x \in E$ and $t, r>0$ where $d_{w} \geq 2$ and $d \geq 1$. Examples satisfying (4.20) include diffusions on fractals such as Sierpinski gaskets and Sierpinski carpets, see for instance [3] and [22] for details and more examples.

Theorem 4.9 We assume that Assumption 4.1 holds. Suppose that the heat kernel of the conservative diffusion process $X$ enjoys estimates (4.20). Let $q(t, x, y)$ be given by (3.2). Then we have the following two statements:

(i) If $\Phi(d(x, y)) \phi\left(t^{-1}\right) \leq 1$, then

$$
q(t, x, y) \simeq \frac{\phi\left(t^{-1}\right)}{t} \int_{\Phi(d(x, y))}^{2 / \phi\left(t^{-1}\right)} \frac{r}{V\left(x, \Phi^{-1}(r)\right)} d r
$$

(ii) If $\Phi(d(x, y)) \phi\left(t^{-1}\right) \geq 1$, then there exist constants $c_{i}>0(i=1, \ldots, 4)$ such that

$$
\begin{aligned}
\frac{c_{1}}{\left.t V\left(x, \Phi^{-1}\left(1 / \phi\left(t^{-1}\right)\right)\right)\right)} & \frac{n(t, d(x, y))}{\phi(n(t, d(x, y)) / t)} \exp \left(-c_{2} n(t, d(x, y))\right) \\
& \leq q(t, x, y) \\
& \leq \frac{c_{3}}{\left.t V\left(x, \Phi^{-1}\left(1 / \phi\left(t^{-1}\right)\right)\right)\right)} \frac{n(t, d(x, y))}{\phi(n(t, d(x, y)) / t)} \exp \left(-c_{4} n(t, d(x, y))\right),
\end{aligned}
$$

where $n(\cdot, r)$ is a non-increasing function on $(0, \infty)$ determined by

$$
\frac{1}{\phi(n(t, r) / t)} \simeq \Phi\left(\frac{r}{n(t, r)}\right), \quad t, r>0
$$


Let us roughly give some intuitive explanations on these estimates between the fundamental solution $q(t, x, y)$ for the time fractional Poisson equation and the fundamental solution $p(r, x, y)$ of the homogenous time fractional equation. According to (3.3),

$$
p(t, x, y)=\int_{0}^{\infty} p^{0}(r, x, y) d_{r} \mathbb{P}\left(E_{t} \leq r\right)=\int_{0}^{\infty} p^{0}(r, x, y) d_{r} \mathbb{P}\left(S_{r} \geq t\right) .
$$

By Proposition 4.7 and Theorem 4.4, for any $t, r>0$,

$$
\bar{p}(r, t) \simeq t^{-1} \mathbb{P}\left(S_{r} \geq t\right) .
$$

Therefore, we shall have the following rough expression

$$
q(t, x, y) \approx \int_{0}^{\infty} p^{0}(r, x, y) \cdot\left(\frac{r}{t}\right) d_{r} \mathbb{P}\left(S_{r} \geq t\right)
$$

that is, to estimate $q(t, x, y)$ we need to add the factor $r / t$ inside the formula above for $p(t, x, y)$. Such intuition is much clear, if we compare Theorem 4.8 (resp. Theorem 4.9) with [13, Theorem 1.6] (resp. [13, Theorem 1.8]). (In particular, for the case that $\Phi(d(x, y)) \phi\left(t^{-1}\right) \leq 1$ or the pure jump case.)

Proof of Theorem 1.4(ii). Again, by the same argument as that in the proof of Theorem 1.4(i), we have $\Phi(r)=r^{\alpha}, V(x, r) \simeq r^{d}$ and $\phi(r)=r^{\beta}$ in this case. For the case $d(x, y) \leq t^{\beta / \alpha}$ (i.e. $\left.\Phi(d(x, y)) \phi\left(t^{-1}\right) \leq 1\right)$, the computation is the same as that of Theorem 1.4(i). For the case $d(x, y) \geq t^{\beta / \alpha}$, according to (4.24),$n=n(t, r)$ is now determined by

$$
\frac{1}{(n / t)^{\beta}} \simeq(r / n)^{\alpha}, \quad t, r>0
$$

hence $n \simeq\left(r^{\alpha} / t^{\beta}\right)^{1 /(\alpha-\beta)}$ (note that $0<\beta<1$ and $\alpha \geq 2$ in this case). Since $n \geq 1$ in this case, we have $n^{1-\beta} \exp (-n) \asymp \exp (-n)$. Given these, the conclusion is an easy consequence of Theorem 4.9 .

\section{Appendix: Proofs of Theorems 4.8 and 4.9}

In this part, we will give proofs of Theorems 4.8 and 4.9, Recall that $\bar{p}^{0}(t, x, d(x, y))$ is defined in (4.18) and (4.20). For simplicity, in the following we fix $x, y \in E$ and let $z=d(x, y)$. Then, $\bar{p}^{0}(t, x, d(x, y))=\bar{p}^{0}(t, x, z)$. Furthermore, we write $\bar{p}^{0}(t, x, z)$ and $V(x, r)$ as $\bar{p}^{0}(t, z)$ and $V(r)$, respectively. According to Theorem 4.4 and (3.2), it holds

$$
\begin{aligned}
q(t, x, y) \asymp & \int_{0}^{2 / \phi\left(t^{-1}\right)} \bar{p}^{0}(r, z) \cdot \frac{1}{t} r \phi\left(t^{-1}\right) d r \\
& +\int_{2 / \phi\left(t^{-1}\right)}^{\infty} \bar{p}^{0}(r, z) \cdot \frac{1}{t} \exp \left(-c_{0} t\left(\phi^{\prime}\right)^{-1}(t / r)\right) d r \\
= & : I_{1}+I_{2} .
\end{aligned}
$$

Proof of Theorem 4.8. The proof is split into two cases. 
Case (1) Suppose that $\Phi(z) \phi\left(t^{-1}\right) \leq 1$. Then

$$
\begin{aligned}
I_{1} & \simeq \frac{\phi\left(t^{-1}\right)}{t}\left(\frac{1}{V(z) \Phi(z)} \int_{0}^{\Phi(z)} r^{2} d r+\int_{\Phi(z)}^{2 / \phi\left(t^{-1}\right)} \frac{r}{V\left(\Phi^{-1}(r)\right)} d r\right) \\
& \simeq \frac{\phi\left(t^{-1}\right)}{t}\left(\frac{\Phi(z)^{2}}{V(z)}+\int_{\Phi(z)}^{2 / \phi\left(t^{-1}\right)} \frac{r}{V\left(\Phi^{-1}(r)\right)} d r\right) \simeq \frac{\phi\left(t^{-1}\right)}{t} \int_{\Phi(z)}^{2 / \phi\left(t^{-1}\right)} \frac{r}{V\left(\Phi^{-1}(r)\right)} d r .
\end{aligned}
$$

Here in the last inequality above we used the fact that

$$
\int_{\Phi(z)}^{2 / \phi\left(t^{-1}\right)} \frac{r}{V\left(\Phi^{-1}(r)\right)} d r \geq \int_{\Phi(z)}^{2 \Phi(z)} \frac{r}{V\left(\Phi^{-1}(r)\right)} d r \geq c \frac{\Phi(z)^{2}}{V(z)}
$$

where the weak scaling properties of $\Phi$ and $V$ are used.

On the other hand, if $\Phi(z) \phi\left(t^{-1}\right) \leq 1$, then by changing variable $s=r \phi\left(t^{-1}\right)$, and using the weak scaling property of $\Phi$ with $\left(\alpha_{1}, \alpha_{2}\right)$, (4.1), (4.17), (4.8) and (4.10),

$$
\begin{aligned}
I_{2} & \simeq \frac{1}{t \phi\left(t^{-1}\right)} \int_{2}^{\infty} \exp \left(-c_{0} t\left(\phi^{\prime}\right)^{-1}\left(t \phi\left(t^{-1}\right) / s\right)\right) \cdot \frac{1}{V\left(\Phi^{-1}\left(s / \phi\left(t^{-1}\right)\right)\right)} d s \\
& =\frac{1}{t \phi\left(t^{-1}\right) V\left(\Phi^{-1}\left(1 / \phi\left(t^{-1}\right)\right)\right)} \int_{2}^{\infty} \exp \left(-c_{0} t\left(\phi^{\prime}\right)^{-1}\left(\phi^{\prime}\left(t^{-1}\right) / s\right)\right) \cdot \frac{V\left(\Phi^{-1}\left(1 / \phi\left(t^{-1}\right)\right)\right)}{V\left(\Phi^{-1}\left(s / \phi\left(t^{-1}\right)\right)\right)} d s \\
& \leq \frac{c}{t \phi\left(t^{-1}\right) V\left(\Phi^{-1}\left(1 / \phi\left(t^{-1}\right)\right)\right)} \sum_{n=1}^{\infty} \int_{2^{n}}^{2^{n+1}} \exp \left(-c_{0} t\left(\phi^{\prime}\right)^{-1}\left(\phi^{\prime}\left(t^{-1}\right) / s\right)\right) s^{-d_{1} / \alpha_{2}} d s \\
& \leq \frac{c}{t \phi\left(t^{-1}\right) V\left(\Phi^{-1}\left(1 / \phi\left(t^{-1}\right)\right)\right)} \sum_{n=1}^{\infty} \exp \left(-c_{0} t\left(\phi^{\prime}\right)^{-1}\left(\phi^{\prime}\left(t^{-1}\right) / 2^{n}\right)\right) 2^{-n\left(\left(d_{1} / \alpha_{2}\right)-1\right)} \\
& \leq \frac{c}{t \phi\left(t^{-1}\right) V\left(\Phi^{-1}\left(1 / \phi\left(t^{-1}\right)\right)\right)} \sum_{n=1}^{\infty} \exp \left(-c_{1} 2^{n\left(1-\beta_{2}\right)}\right) 2^{-n\left(\left(d_{1} / \alpha_{2}\right)-1\right)} \\
& \leq \frac{c}{t \phi\left(t^{-1}\right) V\left(\Phi^{-1}\left(1 / \phi\left(t^{-1}\right)\right)\right)} .
\end{aligned}
$$

Thus

$$
I_{2} \leq \frac{c}{t \phi\left(t^{-1}\right) V\left(\Phi^{-1}\left(1 / \phi\left(t^{-1}\right)\right)\right)}
$$

Note that

$$
\begin{aligned}
\frac{\phi\left(t^{-1}\right)}{t} \int_{\Phi(z)}^{2 / \phi\left(t^{-1}\right)} \frac{r}{V\left(\Phi^{-1}(r)\right)} d r & \geq \frac{\phi\left(t^{-1}\right)}{t} \int_{1 / \phi\left(t^{-1}\right)}^{2 / \phi\left(t^{-1}\right)} \frac{r}{V\left(\Phi^{-1}(r)\right)} d r \\
& \geq \frac{c}{t \phi\left(t^{-1}\right) V\left(\Phi^{-1}\left(1 / \phi\left(t^{-1}\right)\right)\right)} .
\end{aligned}
$$

According to all the estimates above, we know that for any $z, t>0$ with $\Phi(z) \phi\left(t^{-1}\right) \leq 1$,

$$
c_{2} \frac{\phi\left(t^{-1}\right)}{t} \int_{\Phi(z)}^{2 / \phi\left(t^{-1}\right)} \frac{r}{V\left(\Phi^{-1}(r)\right)} d r \leq I_{1} \leq I_{1}+I_{2} \leq c_{3} \frac{\phi\left(t^{-1}\right)}{t} \int_{\Phi(z)}^{2 / \phi\left(t^{-1}\right)} \frac{r}{V\left(\Phi^{-1}(r)\right)} d r .
$$

Case (2) Suppose that $\Phi(z) \phi\left(t^{-1}\right) \geq 1$. Then

$$
I_{1} \simeq \frac{\phi\left(t^{-1}\right)}{t} \int_{0}^{2 / \phi\left(t^{-1}\right)} \frac{r^{2}}{V(z) \Phi(z)} d r \simeq \frac{1}{t \phi\left(t^{-1}\right)^{2} V(z) \Phi(z)} .
$$


On the other hand, following the same argument as (5.3), we find that if $z, t>0$ with $\Phi(z) \phi\left(t^{-1}\right) \geq$ 1 , then

$$
\begin{aligned}
I_{2} & \leq \frac{c}{t \Phi(z) V(z)} \int_{2 / \phi\left(t^{-1}\right)}^{\infty} \exp \left(-c_{0} t\left(\phi^{\prime}\right)^{-1}(t / r)\right) \cdot r d r \\
& \leq \frac{c}{t \phi\left(t^{-1}\right)^{2} \Phi(z) V(z)} \int_{2}^{\infty} \exp \left(-c_{0} t\left(\phi^{\prime}\right)^{-1}\left(\phi^{\prime}\left(t^{-1}\right) / s\right)\right) \cdot s d s \leq \frac{c}{t \phi\left(t^{-1}\right)^{2} \Phi(z) V(z)} .
\end{aligned}
$$

Hence, for any $z, t>0$ with $\Phi(z) \phi\left(t^{-1}\right) \geq 1$,

$$
\frac{c_{1}}{t \phi\left(t^{-1}\right)^{2} V(z) \Phi(z)} \leq I_{1} \leq I_{1}+I_{2} \leq \frac{c_{2}}{t \phi\left(t^{-1}\right)^{2} V(z) \Phi(z)}
$$

The desired estimates for $q(t, x, y)$ now follows from (5.5), (5.6) and (5.1).

Proof of Theorem 4.9, The proof is divided into two cases again.

Case (1) Suppose that $\Phi(z) \phi\left(t^{-1}\right) \leq 1$. Then by (4.23)

$$
\begin{aligned}
I_{1} & \simeq \frac{\phi\left(t^{-1}\right)}{t} \int_{0}^{\Phi(z)} \frac{r}{V\left(\Phi^{-1}(r)\right)} \exp (-m(r, z)) d r+\frac{\phi\left(t^{-1}\right)}{t} \int_{\Phi(z)}^{2 / \phi\left(t^{-1}\right)} \frac{r}{V\left(\Phi^{-1}(r)\right)} d r \\
& =: \frac{\phi\left(t^{-1}\right)}{t}\left(I_{1,1}+I_{1,2}\right) .
\end{aligned}
$$

According to (4.17) and (4.22), we have

$$
\begin{aligned}
I_{1,1} & =\sum_{n=0}^{\infty} \int_{\Phi(z) / 2^{n+1}}^{\Phi(z) / 2^{n}} \frac{r}{V\left(\Phi^{-1}(r)\right)} \exp (-m(r, z)) d r \\
& \leq \sum_{n=0}^{\infty} \frac{\left(\Phi(z) / 2^{n}\right)^{2}}{V\left(\Phi^{-1}\left(\Phi(z) / 2^{n+1}\right)\right)} \exp \left(-m\left(\Phi(z) / 2^{n}, z\right)\right) \\
& \leq \frac{c \Phi(z)^{2}}{V(z)} \sum_{n=0}^{\infty} 2^{n\left(\left(d_{2} / \alpha_{1}\right)-2\right)} \exp \left(-c_{1} m(\Phi(z), z) 2^{n /\left(\alpha_{2}-1\right)}\right) \leq \frac{c \Phi(z)^{2}}{V(z)},
\end{aligned}
$$

where in the last inequality we used (4.23). This estimate along with (5.2) yields that

$$
I_{1} \simeq \frac{\phi\left(t^{-1}\right)}{t} \int_{\Phi(z)}^{2 / \phi\left(t^{-1}\right)} \frac{r}{V\left(\Phi^{-1}(r)\right)} d r .
$$

On the other hand, if $\Phi(z) \phi\left(t^{-1}\right) \leq 1$, then, by the argument of (5.3),

$$
I_{2} \leq \frac{c}{t} \int_{2 / \phi\left(t^{-1}\right)}^{\infty} \exp \left(-c_{0} t\left(\phi^{\prime}\right)^{-1}(t / r)\right) \cdot \frac{1}{V\left(\Phi^{-1}(r)\right)} d r \leq \frac{c}{t \phi\left(t^{-1}\right) V\left(\Phi^{-1}\left(1 / \phi\left(t^{-1}\right)\right)\right)} .
$$

We have by (5.4) that

$$
I_{1}+I_{2} \leq \frac{c \phi\left(t^{-1}\right)}{t} \int_{\Phi(z)}^{2 / \phi\left(t^{-1}\right)} \frac{r}{V\left(\Phi^{-1}(r)\right)} d r .
$$

Therefore, for any $z, t>0$ satisfying $\Phi(z) \phi\left(t^{-1}\right) \leq 1$, it holds that

$$
\frac{c_{1} \phi\left(t^{-1}\right)}{t} \int_{\Phi(z)}^{2 / \phi\left(t^{-1}\right)} \frac{r}{V\left(\Phi^{-1}(r)\right)} d r \leq I_{1} \leq I_{1}+I_{2} \leq \frac{c_{2} \phi\left(t^{-1}\right)}{t} \int_{\Phi(z)}^{2 / \phi\left(t^{-1}\right)} \frac{r}{V\left(\Phi^{-1}(r)\right)} d r .
$$


Case (2) Suppose that $\Phi(z) \phi\left(t^{-1}\right) \geq 1$. Then by (4.17) and (4.22), we have

$$
\begin{aligned}
I_{1} & \leq \frac{c \phi\left(t^{-1}\right)}{t} \int_{0}^{2 / \phi\left(t^{-1}\right)} \frac{r}{V\left(\Phi^{-1}(r)\right)} \exp (-m(r, z)) d r \\
& =\frac{c \phi\left(t^{-1}\right)}{t} \sum_{n=0}^{\infty} \int_{2^{1-n} / \phi\left(t^{-1}\right)}^{2^{-n} / \phi\left(t^{-1}\right)} \frac{r}{V\left(\Phi^{-1}(r)\right)} \exp (-m(r, z)) d r \\
& \leq \frac{c \phi\left(t^{-1}\right)}{t} \sum_{n=0}^{\infty} \frac{\left(2^{-n} / \phi\left(t^{-1}\right)\right)^{2}}{V\left(\Phi^{-1}\left(2^{1-n} / \phi\left(t^{-1}\right)\right)\right)} \exp \left(-m\left(2^{-n} / \phi\left(t^{-1}\right), z\right)\right) \\
& \leq \frac{c}{t \phi\left(t^{-1}\right) V\left(\Phi^{-1}\left(1 / \phi\left(t^{-1}\right)\right)\right)} \sum_{n=0}^{\infty} 2^{n\left(\left(d_{2} / \alpha_{1}\right)-2\right)} \exp \left(-c_{1} m\left(1 / \phi\left(t^{-1}\right), z\right) 2^{n /\left(\alpha_{2}-1\right)}\right) \\
& \leq \frac{c}{t \phi\left(t^{-1}\right) V\left(\Phi^{-1}\left(1 / \phi\left(t^{-1}\right)\right)\right)} \exp \left(-c_{1} m\left(1 / \phi\left(t^{-1}\right), z\right)\right)
\end{aligned}
$$

where in the last inequality we used the facts that the function $r \mapsto m(r, z)$ is non-increasing and (4.23) so that

$$
\begin{aligned}
\exp \left(-c_{1} m\left(1 / \phi\left(t^{-1}\right), z\right)\left(2^{n /\left(\alpha_{2}-1\right)}-1\right)\right) & \leq \exp \left(-c_{1} m(\Phi(z), z)\left(2^{n /\left(\alpha_{2}-1\right)}-1\right)\right) \\
& \leq \exp \left(-c_{2}\left(2^{n /\left(\alpha_{2}-1\right)}-1\right)\right) .
\end{aligned}
$$

To get the estimate for $I_{2}$, we need to consider the following two functions inside the estimates of $\bar{p}(r, t)$ and the exponential terms of $\bar{p}^{0}(r, z)$ respectively:

$$
G_{1}(r)=t\left(\phi^{\prime}\right)^{-1}(t / r) \quad \text { and } \quad G_{2}(r)=m(r, z)
$$

for all $r>0$ and fixed $z, t>0$. Note that, by (4.10), (4.22) and the facts that $\phi^{\prime}$ and $m(\cdot, z)$ are non-increasing on $(0, \infty), G_{1}(r)$ is a non-decreasing function on $(0, \infty)$ such that $G_{1}(0)=0$ and $G_{1}(\infty)=\infty$, and $G_{2}(r)$ is a non-increasing function on $(0, \infty)$ such that $G_{2}(0)=\infty$ and $G_{2}(\infty)=0$. Thus, noting that $m(r, z)$ satisfies (4.22), there exist $c^{*}, c_{*}>0$ and $r_{0}=r_{0}(z, t) \in(0, \infty)$ such that $G_{1}\left(r_{0}\right) \simeq G_{2}\left(r_{0}\right), G_{1}(r) \geq c^{*} G_{2}(r)$ when $r \geq r_{0}$, and $G_{1}(r) \leq c_{*} G_{2}(r)$ when $r \leq r_{0}$.

On the other hand, since $\Phi(z) \phi\left(t^{-1}\right) \geq 1$, by (4.8), (4.23) and the fact that $m(\cdot, z)$ is nonincreasing on $(0, \infty)$,

$$
\begin{aligned}
G_{1}\left(1 / \phi\left(t^{-1}\right)\right) & =t\left(\phi^{\prime}\right)^{-1}\left(t \phi\left(t^{-1}\right)\right) \simeq t\left(\phi^{\prime}\right)^{-1}\left(\phi^{\prime}\left(t^{-1}\right)\right)=1 \\
& \leq c_{3} m(\Phi(z), z) \leq c_{1} m\left(1 / \phi\left(t^{-1}\right), z\right)=c_{1} G_{2}\left(1 / \phi\left(t^{-1}\right)\right)
\end{aligned}
$$

and

$$
G_{1}(\Phi(z)) \geq G_{1}\left(1 / \phi\left(t^{-1}\right)\right) \simeq t\left(\phi^{\prime}\right)^{-1}\left(\phi^{\prime}\left(t^{-1}\right)\right)=1 \geq c_{4} m(\Phi(z), z)=c_{2} G_{2}(\Phi(z)),
$$

where constants $c_{3}, c_{4}$ are independent of $t$ and $z$. Hence there are constants $c_{5}, c_{6}>0$ independent of $t$ and $z$ such that

$$
\frac{2}{\phi\left(t^{-1}\right)} \leq c_{5} r_{0} \leq c_{6} \Phi(z)
$$


Combining all the estimates above, we find that

$$
\begin{aligned}
I_{2}= & \frac{1}{t} \int_{2 / \phi\left(t^{-1}\right)}^{\infty} \exp \left(-c_{0} t\left(\phi^{\prime}\right)^{-1}(t / r)\right) \cdot \bar{p}^{0}(r, z) d r \\
\leq & \frac{1}{t V\left(\Phi^{-1}\left(2 / \phi\left(t^{-1}\right)\right)\right)} \int_{2 / \phi\left(t^{-1}\right)}^{\infty} \exp \left(-c_{0} t\left(\phi^{\prime}\right)^{-1}(t / r)\right) \cdot \exp (-m(r, z)) d r \\
\leq & \frac{c}{t V\left(\Phi^{-1}\left(1 / \phi\left(t^{-1}\right)\right)\right)} \int_{2 / \phi\left(t^{-1}\right)}^{c_{5} r_{0}} \exp (-m(r, z)) d r \\
& +\frac{c}{t V\left(\Phi^{-1}\left(1 / \phi\left(t^{-1}\right)\right)\right)} \int_{c_{5} r_{0}}^{\infty} \exp \left(-c_{0} t\left(\phi^{\prime}\right)^{-1}(t / r)\right) d r \\
= & : \frac{c}{t V\left(\Phi^{-1}\left(1 / \phi\left(t^{-1}\right)\right)\right)} I_{2,1}+\frac{c}{t V\left(\Phi^{-1}\left(1 / \phi\left(t^{-1}\right)\right)\right)} I_{2,2} .
\end{aligned}
$$

According to (4.22),

$$
\begin{aligned}
I_{2,1} & \leq \int_{0}^{c_{5} r_{0}} \exp (-m(r, z)) d r=\sum_{n=0}^{\infty} \int_{c_{5} r_{0} / 2^{n+1}}^{c_{5} r_{0} / 2^{n}} \exp (-m(r, z)) d r \\
& \leq c r_{0} \sum_{n=0}^{\infty} 2^{-n} \cdot \exp \left(-m\left(c_{5} r_{0} / 2^{n}, z\right)\right) \leq c r_{0} \sum_{n=0}^{\infty} 2^{-n} \cdot \exp \left(-c_{6} m\left(r_{0}, z\right) 2^{n /\left(\alpha_{2}-1\right)}\right) \\
& \leq c r_{0} \exp \left(-c_{6} G_{2}\left(r_{0}\right)\right)
\end{aligned}
$$

where in the last inequality we used the facts that the function $r \mapsto m(r, z)$ is non-increasing and

$$
\begin{aligned}
\sum_{n=0}^{\infty} 2^{-n} \cdot \exp \left(-c_{6} m\left(r_{0}, z\right)\left(2^{n /\left(\alpha_{2}-1\right)}-1\right)\right) & \leq \sum_{n=0}^{\infty} 2^{-n} \cdot \exp \left(-c_{7} m(\Phi(z), z)\left(2^{n /\left(\alpha_{2}-1\right)}-1\right)\right) \\
& \leq \sum_{n=0}^{\infty} 2^{-n} \cdot \exp \left(-c_{8}\left(2^{n /\left(\alpha_{2}-1\right)}-1\right)\right) \leq c_{9},
\end{aligned}
$$

thanks to (5.11). On the other hand, by (4.9),

$$
\begin{aligned}
I_{2,2} & =\sum_{n=0}^{\infty} \int_{2^{n} c_{5} r_{0}}^{2^{n+1} c_{5} r_{0}} \exp \left(-c_{0} t\left(\phi^{\prime}\right)^{-1}(t / r)\right) d r \\
& \leq c r_{0} \sum_{n=0}^{\infty} 2^{n} \cdot \exp \left(-c_{0} t\left(\phi^{\prime}\right)^{-1}\left(t /\left(2^{n} c_{5} r_{0}\right)\right) \leq c r_{0} \sum_{n=0}^{\infty} 2^{n} \cdot \exp \left(-c_{10} t\left(\phi^{\prime}\right)^{-1}\left(t / r_{0}\right) 2^{n\left(1-\beta_{2}\right)}\right)\right. \\
& \leq c r_{0} \exp \left(-c_{10} t\left(\phi^{\prime}\right)^{-1}\left(t / r_{0}\right)\right)=c r_{0} \exp \left(-c_{10} G_{1}\left(r_{0}\right)\right) \leq c r_{0} \exp \left(-c_{11} G_{2}\left(r_{0}\right)\right),
\end{aligned}
$$

where in the last inequality we used the facts that the function $r \mapsto m(r, z)$ is non-increasing and

$$
\begin{aligned}
\sum_{n=0}^{\infty} 2^{n} \exp \left(-c_{10} t\left(\phi^{\prime}\right)^{-1}\left(t / r_{0}\right)\left(2^{n\left(1-\beta_{2}\right)}-1\right)\right) & \leq \sum_{n=0}^{\infty} 2^{n} \exp \left(-c_{10} t\left(\phi^{\prime}\right)^{-1}\left(c_{12} t \phi\left(t^{-1}\right)\right)\left(2^{n\left(1-\beta_{2}\right)}-1\right)\right) \\
& \leq \sum_{n=0}^{\infty} 2^{n} \exp \left(-c_{13}\left(2^{n\left(1-\beta_{2}\right)}-1\right)\right) \leq c_{14},
\end{aligned}
$$

thanks to (5.11) again. Putting these estimates together, we obtain

$$
I_{2} \leq \frac{c}{t V\left(\Phi^{-1}\left(1 / \phi\left(t^{-1}\right)\right)\right)} r_{0} \exp \left(-\left(c_{6} \wedge c_{11}\right) G_{2}\left(r_{0}\right)\right) .
$$


Next, we rewrite the exponential term in the right hand side of (5.12). By the fact that $m\left(r_{0}, z\right)=G_{2}\left(r_{0}\right) \asymp G_{1}\left(r_{0}\right)=t\left(\phi^{\prime}\right)^{-1}\left(t / r_{0}\right)$ and (4.21), we have

$$
\frac{r_{0}}{t\left(\phi^{\prime}\right)^{-1}\left(t / r_{0}\right)} \simeq \Phi\left(\frac{z}{t\left(\phi^{\prime}\right)^{-1}\left(t / r_{0}\right)}\right) \text {. }
$$

Let $s_{0}=\left(\phi^{\prime}\right)^{-1}\left(t / r_{0}\right)$. Then $t / r_{0}=\phi^{\prime}\left(s_{0}\right)$ and, by (4.8),

$$
\frac{1}{\phi\left(\left(t s_{0}\right) / t\right)}=\frac{1}{\phi\left(s_{0}\right)} \simeq \frac{1}{\phi^{\prime}\left(s_{0}\right) s_{0}} \simeq \Phi\left(\frac{z}{t s_{0}}\right) .
$$

Thus, (5.12) and (5.13) yield that

$$
I_{2} \leq \frac{c}{t V\left(\Phi^{-1}\left(1 / \phi\left(t^{-1}\right)\right)\right)} r_{0} \exp \left(-c_{15} n(t, z)\right)
$$

where $n(t, z):=m\left(r_{0}, z\right)$ satisfies

$$
\frac{1}{\phi(n(t, z) / t)} \simeq \Phi\left(\frac{z}{n(t, z)}\right)
$$

Since $t\left(\phi^{\prime}\right)^{-1}\left(t / r_{0}\right) \simeq n(t, z)$,

$$
r_{0} \simeq t / \phi^{\prime}(n(t, z) / t) \simeq n(t, z) / \phi(n(t, z) / t),
$$

and so, we have

$$
I_{2} \leq \frac{c n(t, z)}{t \phi(n(t, z) / t) V\left(\Phi^{-1}\left(1 / \phi\left(t^{-1}\right)\right)\right)} \exp \left(-c_{15} n(t, z)\right) .
$$

Following the argument above, we can also obtain that

$$
I_{2} \geq \frac{c n(t, z)}{t \phi(n(t, z) / t) V\left(\Phi^{-1}\left(1 / \phi\left(t^{-1}\right)\right)\right)} \exp \left(-c_{16} n(t, z)\right) .
$$

Set $n:=n(t, z)$. By (5.11), we have $n=G_{2}\left(r_{0}\right) \geq m\left(c_{5}^{-1} c_{6} \Phi(z), z\right) \simeq 1$. Hence, $1 / \phi\left(t^{-1}\right) \leq$ $\mathrm{cn} / \phi(n / t)$ for any $t>0$. Using this, (5.9) and (5.11) again, we get

$$
\begin{aligned}
I_{1} & \leq \frac{c_{17}}{t \phi\left(t^{-1}\right) V\left(\Phi^{-1}\left(1 / \phi\left(t^{-1}\right)\right)\right)} \exp \left(-c_{1} m\left(1 / \phi\left(t^{-1}\right), z\right)\right) \\
& \leq \frac{c_{18} n}{t \phi(n / t) V\left(\Phi^{-1}\left(1 / \phi\left(t^{-1}\right)\right)\right)} \exp \left(-c_{19} n\right) .
\end{aligned}
$$

By (5.14) - (5.16), we obtain that for any $z, t>0$ with $\Phi(z) \phi\left(t^{-1}\right) \geq 1$

$$
\begin{aligned}
\frac{c_{20} n}{t \phi(n / t) V\left(\Phi^{-1}\left(1 / \phi\left(t^{-1}\right)\right)\right)} \exp \left(-c_{21} n\right) & \leq I_{2} \leq I_{1}+I_{2} \\
& \leq \frac{c_{22} n}{t \phi(n / t) V\left(\Phi^{-1}\left(1 / \phi\left(t^{-1}\right)\right)\right)} \exp \left(-c_{23} n\right) .
\end{aligned}
$$

Therefore, combining (5.8), (5.17) and (5.1), we get the desired estimates for $p(t, x, y)$. 
Acknowledgements. The research of Zhen-Qing Chen is partially supported by Simons Foundation Grant 520542 and a Victor Klee Faculty Fellowship at UW. The research of Panki Kim is supported by the National Research Foundation of Korea (NRF) grant funded by the Korea government (MSIP) (No. 2016R1E1A1A01941893). The research of Takashi Kumagai is supported by JSPS KAKENHI Grant Number JP17H01093 and by the Alexander von Humboldt Foundation. The research of Jian Wang is supported by the National Natural Science Foundation of China (Nos. 11522106 and 11831014), the Fok Ying Tung Education Foundation (No. 151002), the Program for Probability and Statistics: Theory and Application (No. IRTL1704) and the Program for Innovative Research Team in Science and Technology in Fujian Province University (IRTSTFJ).

The main results of this paper, including the two-sided estimates on the fundamental solution $q(t, x, y)$ for the time fractional Poisson equations, have been reported in several conferences including Fractional PDEs: theory, algorithms and applications held from June 18 to 22, 2018 at Brown University, Providence, The Fifth IMS Asia Pacific Rim Meeting from June 26 to 29, 2018 in Singapore, Workshop on Dynamics, Control and Numerics for Fractional PDEs from December 5 to 7, 2018 at San Juan, Puerto Rico, and Non Standard Diffusions in Fluids, Kinetic Equations and Probability from December 10 to 14, 2018 at CIRM, Marseille. We thank the organizers for the invitations and participants for their interest.

\section{References}

[1] M. Allen, L. Caffarelli and A. Vasseur, A parabolic problem with a fractional time derivative. Arch. Ration. Mech. Anal. 221 (2016), 603-630.

[2] D.G. Aronson, Bounds on the fundamental solution of a parabolic equation. Bull. Amer. Math. Soc. 73, (1967), 890-896.

[3] M.T. Barlow, Diffusions on Fractals. Lect. Notes Math. 1690, Ecole d'été de probabilités de Saint-Flour XXV-1995, Springer, New York, 1998.

[4] M.T. Barlow, T. Coulhon and T. Kumagai, Characterization of sub-Gaussian heat kernel estimates on strongly recurrent graphs, Comm. Pure Appl. Math. 58 (2005), 1642-1677.

[5] M.T. Barlow and E.A. Perkins, Brownian motion on the Sierpinski gasket. Probab. Theory Relat. Fields 79, (1988), 543-623.

[6] B. Baeumer and M.M. Meerchaert, Stochastic solutions for fractional Cauchy problems. Fract. Calc. Appl. Anal. 4 (2001), 481-500.

[7] J. Bertoin, Lévy Processes. Cambridge University Press, Cambridge, 1996.

[8] Z.-Q. Chen, Time fractional equations and probabilistic representation. Chaos, Solitons and Fractals 102 (2017), 168-174.

[9] Z.-Q. Chen and T. Kumagai, Heat kernel estimates for stable-like processes on $d$-sets. Stoch. Proc. Appl. 108 (2003), 27-62.

[10] Z.-Q. Chen and T. Kumagai, Heat kernel estimates for jump processes of mixed types on metric measure spaces. Probab. Theory Relat. Fields 140 (2008), 277-317.

[11] Z.-Q. Chen, T. Kumagai and J. Wang, Stability of heat kernel estimates for symmetric non-local Dirichlet forms on metric measure spaces, to appear in Memoirs Amer. Math. Soc. 
[12] Z.-Q. Chen, K.-H. Kim and P. Kim, Fractional time stochastic partial differential equations. Stoch. Proc. Appl. 125 (2015), 1470-1499.

[13] Z.-Q. Chen, P. Kim, T. Kumagai and J. Wang, Heat kernel estimates for solutions of general time fractional equations. Forum Math. 30 (2018), 1163-1192.

[14] Q. Du, L. Toniazzi and Z. Zhou, Stochastic representation of solution to nonlocal-in-time diffusion. arXiv:1810.08788 [math.AP]

[15] S.D. Eidelman, S.D. Ivasyshen and A.N. Kochubei, Analytic Methods in the Theory of Differential and Pseudo-Differential Equations of Parabolic Type. Operator Theory: Advances and Applications 152, Springer Basel, Switzerland, 2004.

[16] S.D. Eidelman, and A.N. Kochubei, Cauchy problem for fractional diffusion equations. J. Differ. Equ. 199 (2004), 211-255.

[17] M. Foondun, and E. Nane, Asymptotic properties of some space-time fractional stochastic equations. Math. Z. 287 (2017), 493-519.

[18] M. Grothaus and F. Jahnert, Mittag-Leffler analysis II: Application to the fractional heat equation. $J$. Funct. Anal. 270 (2016), 2732-2768.

[19] A. Grigor'yan, Heat kernels and function theory on metric measure spaces. Cont. Math. 338 (2003), $143-172$.

[20] A. Grigor'yan, J. Hu, and K.-S. Lau, Heat kernels on metric measure spaces and an application to semilinear elliptic equations. Trans. Amer. Math. Soc. 355 (2003), 2065-2095.

[21] A. Grigor'yan and A. Telcs, Two-sided estimates of heat kernels on metric measure spaces. Annals of Probability 40 (2012), 1212-1284.

[22] B.M. Hambly and T. Kumagai, Transition density estimates for diffusion processes on post critically finite self-similar fractals. Proc. London Math. Soc. 78 (1999), 431-458.

[23] P. Hartman and A. Wintner, On the infinitesimal generators of integral convolutions. Amer. J. Math. 64 (1942), 273-298.

[24] M. E. Hernández-Hernández and V. N. Kolokoltsov, On the solution of two-sided fractional ordinary differential equations of Caputo type. Fract. Calc. Appl. Anal. 19 (2016), 1393-1413.

[25] M. E. Hernández-Hernández, V. N. Kolokoltsov and L. Toniazzi, Generalised fractional evolution equations of Caputo type. Chaos, Solitons and Fractals 102 (2017), 184-196.

[26] P. Kim, R. Song and Z. Vondraček, Global uniform boundary Hanarck principle with explicit decay rate and its application. Stoch. Proc. Appl. 124 (2014), 235-267.

[27] V. N. Kolokoltsov, On fully mixed and multidimensional extensions of the Caputo and RiemannLiouville derivatives, related Markov processes and fractional differential equations. Fract. Calc. Appl. Anal. 18 (2015), 1039-1073.

[28] K. Li, J. Peng and J. Jia, Cauchy problems for fractional differential equations with Riemann-Liouville fractional derivatives. J. Funct. Anal. 263 (2012), 476-510.

[29] M.M. Meerschaert and H.-P. Scheffler, Stochastic model for ultraslow diffusion. Stoch. Proc. Appl. 116 (2006), 1215-1235.

[30] M.M. Meerschaert and A. Sikorskii, Stochastic Models for Fractional Calculus. De Gruyter Studies in Mathematics 43, Walter de Gruyter, Berlin/Boston, 2012. 
[31] J. Mijena and E. Nane, Space time fractional stochastic partial differential equations. Stoch. Proc. Appl. 125 (2015), 3301-3326.

[32] K.S. Miller and B. Ross, An Introduction to the Fractional Calculus and Fractional Differential Equations. John Wiley and Sons, New York, 1993.

[33] S.G. Samko, A.A. Kilbas and O.I. Marichev, Fractional Integrals and Derivatives: Theory and Applications. Gordon and Breach Science Publishers, New York and London, 1993.

[34] K.-I., Sato and M. Yamazato, On distribution functions of class L. Z. Wahrsch. Verw. Gebiete 43 (1978), 273-308.

[35] K.-I. Sato, Lévy Processes and Infinitely Divisible Distributions. Cambridge University Press, Cambridge, 1999.

[36] R. L. Schilling, R. Song and Z. Vondraček, Bernstein Functions. Theory and Applications (2nd Edn). De Gruyter Studies in Mathematics 37, Walter de Gruyter, Berlin, 2012.

[37] L. Toniazzi, Stochastic solutions for space-time fractional evolution equations on bounded domain. $J$. Math. Anal. Appl. 469 (2019), 594-622

[38] S. Umarov, On fractional Duhamel's principle and its applications. J. Differ. Equ. 252 (2012), 52175234 .

[39] S. Umarov and E. Saydamatov, A fractional analog of the Duhamel principle. Fract. Calc. Appl. Anal. 9 (2006), 57-70.

[40] S. J. Wolfe, On the unimodality of $L$ functions. Ann. Math. Statist. 42 (1971), 912-918.

[41] V.V. Uchaikin, Fractional Derivatives for Physicists and Engineers. Volume I. Background and Theory. Springer \& Higher Education Press, Berlin \& Beijing, 2013.

[42] V.V. Uchaikin, Fractional Derivatives for Physicists and Engineers. Volume II. Applications. Springer \& Higher Education Press, Berlin \& Beijing, 2013.

[43] R. Zacher, Weak solutions of abstract evolutionary integro-differential equations in Hilbert spaces. Funkcial. Ekvac. 52 (2009), 1-18.

\section{Zhen-Qing Chen}

Department of Mathematics, University of Washington, Seattle, WA 98195, USA

E-mail: zqchen@uw.edu

\section{Panki Kim}

Department of Mathematical Sciences and Research Institute of Mathematics, Seoul National University, Building 27, 1 Gwanak-ro,

Gwanak-gu, Seoul 08826, Republic of Korea

E-mail: pkim@snu.ac.kr

\section{Takashi Kumagai}

Research Institute for Mathematical Sciences, Kyoto University, Kyoto 606-8502, Japan

E-mail: kumagai@kurims.kyoto-u.ac.jp

\section{Jian Wang}

College of Mathematics and Informatics \& Fujian Key Laboratory of Mathematical

Analysis and Applications (FJKLMAA), Fujian Normal University, 350007 Fuzhou, P.R. China. E-mail: jianwang@fjnu.edu.cn 